\title{
Thoracic Imaging at Exacerbation of Chronic Obstructive Pulmonary Disease: A Systematic Review
}

This article was published in the following Dove Press journal: International Journal of Chronic Obstructive Pulmonary Disease

\author{
Bojidar A Rangelov $\mathbb{D}^{\prime}$ \\ Alexandra L Young ${ }^{1-3}$ \\ Joseph Jacob ${ }^{1,4}$ \\ Anthony P Cahn ${ }^{5}$ \\ Sarah Lee $\mathbb{1}^{6}$ \\ Frederick J Wilson (iD ${ }^{5}$ \\ David J Hawkes (D) \\ John R Hurst ${ }^{4}$ \\ 'Centre for Medical Image Computing, \\ Department of Medical Physics and \\ Biomedical Engineering, University \\ College London, London, UK; \\ ${ }^{2}$ Department of Computer Science, \\ University College London, London, UK; \\ ${ }^{3}$ Department of Neuroimaging, Institute \\ of Psychiatry, Psychology and \\ Neuroscience, King's College London, \\ London, UK; ${ }^{4} \mathrm{UCL}$ Respiratory, \\ University College London, London, UK; \\ ${ }^{5}$ GlaxoSmithKline Research and \\ Development, Stevenage, UK; ${ }^{6}$ Amallis \\ Consulting LTD, London, UK
}

\begin{abstract}
Exacerbations of chronic obstructive pulmonary disease (COPD) are currently diagnosed based on changes in respiratory symptoms. Characterizing the imaging manifestation of exacerbations could be useful for objective diagnosis of exacerbations in the clinic and clinical trials, as well as provide a mechanism for monitoring exacerbation treatment and recovery. In this systematic review, we employed a comprehensive search across three databases (Medline, EMBASE, Web of Science) to identify studies that performed imaging of the thorax at COPD exacerbation. We included 51 from a total of 5,047 articles which met all our inclusion criteria. We used an adapted version of the Modified Newcastle-Ottawa Quality Assessment Scale for cohort studies to assess the quality of the included studies. Conclusions were weighted towards higher-quality articles. We identified a total of 36 thoracic imaging features studied at exacerbation of COPD. Studies were generally heterogeneous in their measurements and focus. Nevertheless, considering studies which performed consecutive imaging at stable state and exacerbation, which scored highest for quality, we identified salient imaging biomarkers of exacerbations. An exacerbation is characterized by airway wall and airway calibre changes, hyperinflation, pulmonary vasoconstriction and imaging features suggestive of pulmonary arterial hypertension. Most information was gained from CT studies. We present the first ever composite imaging signature of COPD exacerbations. While imaging during an exacerbation is comparatively new and not comprehensively studied, it may uncover important insights into the acute pathophysiologic changes in the cardiorespiratory system during exacerbations of COPD, providing objective confirmation of events and a biomarker of recovery and treatment response.
\end{abstract}

Keywords: COPD, radiology and other imaging, emphysema

\section{Introduction}

Exacerbations of chronic obstructive pulmonary disease (COPD) impose a great burden on patients' quality of life and healthcare systems. In addition to causing significant increases in mortality and disease progression, exacerbations of COPD amount to $\$ 18$ billion in direct costs annually, as well as further spending associated with care and losses in productivity. ${ }^{1}$ According to the Global initiative for Chronic Obstructive Lung Disease (GOLD), an exacerbation is "an episode characterized by an acute worsening of respiratory symptoms which results in additional therapy."2 Reducing the frequency and severity of exacerbations is a major goal in the management of COPD.

Despite the importance of exacerbations, our understanding of these events is incomplete. In particular, the current definition of an exacerbation, being grounded
Correspondence: John R Hurst Email j.hurst@ucl.ac.uk
International Journal of Chronic Obstructive Pulmonary Disease 2020:15 175I-1787

$175 \mid$

DovePress $f$ in $\boldsymbol{v}$ 
solely in a change in symptoms, does not take into account underlying structural and physiological changes that occur in the lung. A worsening of respiratory symptoms may, alternatively or additionally, be caused by other co-morbid conditions. Thus, in clinical practice, exacerbation is a clinical diagnosis of exclusion - if a patient presents with worsening respiratory symptoms and if no alternative conditions are diagnosed then the patient is classified as having an exacerbation. Medical imaging may, therefore, provide the means to inform care for exacerbation in three ways. First, it could enable a "positive" diagnosis of exacerbation, which could diminish misdiagnosis due to comorbidities and facilitate quantification of the effects of novel drugs during clinical trials. Second, identifying relevant changes in the lung at exacerbation is a step towards an improved understanding of pathophysiology and might motivate the development of new therapies. Finally, discovery of rigorous imaging biomarkers of exacerbation could lay the foundations for exacerbation phenotyping.

In this work, we systematically review the available research in which thoracic imaging has been performed at exacerbation of COPD, to determine which imaging biomarkers are characteristic of an exacerbation. There is no prior systematic review of imaging biomarkers at exacerbation of COPD.

\section{Patients and Methods}

\section{Study Population}

We searched for studies including patients with COPD experiencing an exacerbation during which imaging of the thorax was performed. Studies that discussed imaging features that predict or correlate with future exacerbation risk but did not perform imaging at exacerbation were excluded. Studies that enrolled COPD patients for undifferentiated acute respiratory episodes were considered (even if there was no explicit definition of exacerbation), but only when they employed consecutive enrolment. Such studies were included in order to ensure we captured cases which were presenting with potential exacerbations. Studies which selectively enrolled for alternative conditions such as pulmonary embolism and pneumonia were excluded.

Both cross-sectional studies, which performed imaging only at exacerbation, and longitudinal studies, which performed imaging at additional time-points either before or after an exacerbation were included.

\section{Literature Search}

The search strategy was developed in accordance with the Preferred Reporting Items for Systematic Reviews and Meta-Analyses (PRISMA) guidelines ${ }^{3}$ and the $\mathrm{PICO} /$ PECO framework ${ }^{4}$ with the following fields:

- Problem: Chronic Obstructive Pulmonary Disease (COPD)

- Exposure: Exacerbation of COPD

- Comparison: Stable COPD

- Outcomes: Catalogue of imaging features ("biomarkers") which characterize an exacerbation.

We employed a broad search strategy. Three databases were searched up to November 24, 2019 - Medline, EMBASE and Web of Science. We used text terms, Medical Subject Headings (MeSH) terms in Medline and Emtree terms in EMBASE. The terms we used can be grouped into three categories: terms describing COPD, terms describing exacerbations and terms describing imaging. We matched the search strategies in each of the three databases as closely as possible. There were no filters imposed on the searches in order to maximize sensitivity.

The systematic review was registered on the International Prospective Register for Systematic Reviews - PROSPERO (Unique ID: CRD42018095417) ${ }^{5}$ before the start of data extraction. The type of articles included were all original research studies. We did not find any existing systematic reviews on the topic. Reviews, editorials, letters, opinions or conference abstracts and proceedings were excluded. Only articles in English and studies in human subjects were included. For the purposes of this review, imaging was defined as encompassing the typical clinical imaging modalities (CT, X-ray, MRI, Ultrasound, PET, SPECT, etc.), but not microscopy or direct cell imaging techniques.

The review proceeded by first combining and deduplicating search results in the software package EndNote, Philadelphia, PA, USA. ${ }^{6}$ Further to this, the deduplicated results were exported to the online systematic review platform Rayyan, Doha, Qatar, ${ }^{7}$ where screening of titles and abstracts was independently performed by two authors (BAR and JRH). The authors were blinded to each other's decisions. At this stage, further identification and removal of duplicate records was performed (Figure 1). Upon completion of screening, conflicts between the two authors were resolved by reading the complete papers and 

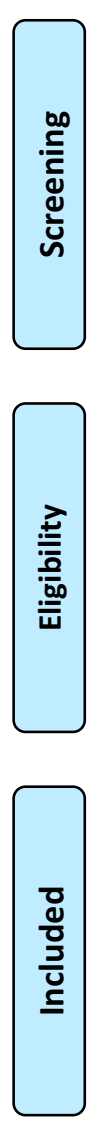

Records identified through database searching $(n=6,718)$
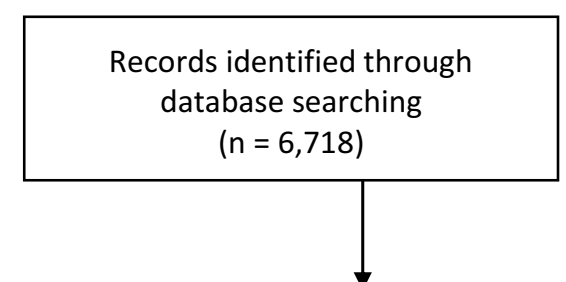

Records after duplicates removed

( $n=5,047)$
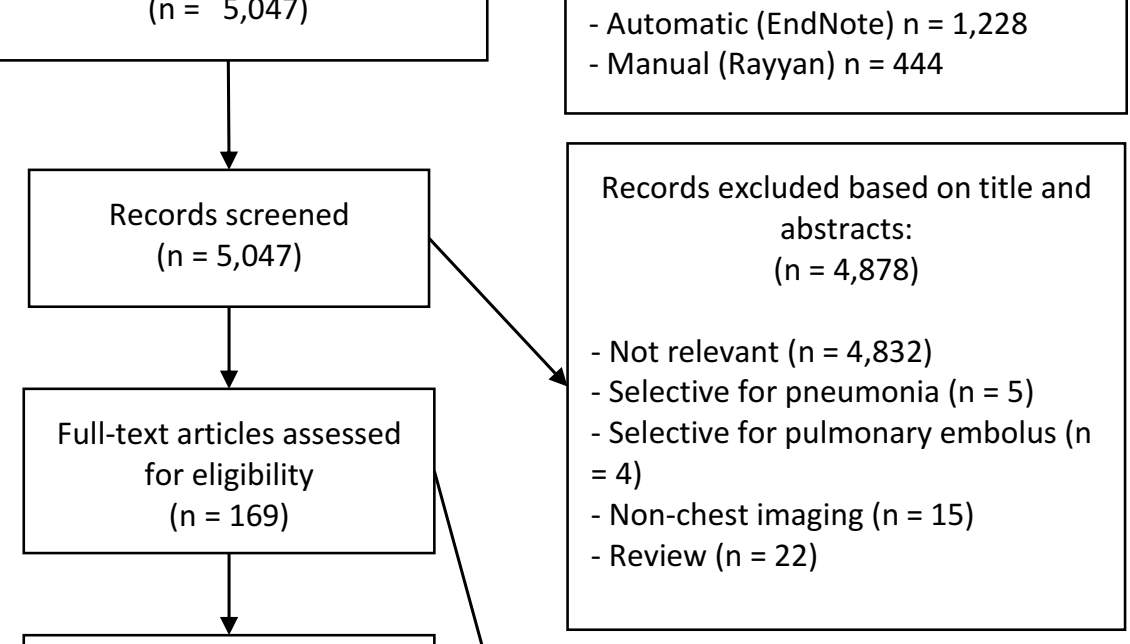

Studies included in qualitative synthesis $(n=51)$

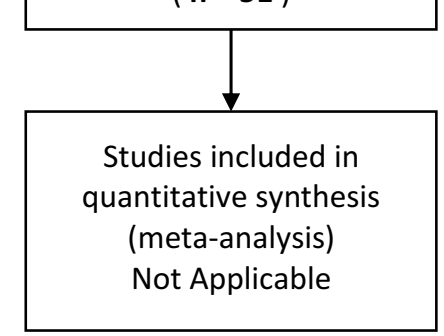

Additional records identified through other sources $(n=1)$
Duplicates removed:

$(n=1,672)$

- Automatic (EndNote) $\mathrm{n}=1,228$

- Manual (Rayyan) $n=444$

Records excluded based on title and abstracts:

( $n=4,878)$

- Not relevant ( $n=4,832$ )

- Selective for pneumonia $(n=5)$

- Selective for pulmonary embolus ( $n$

-4)

-chest imaging $(n=15)$

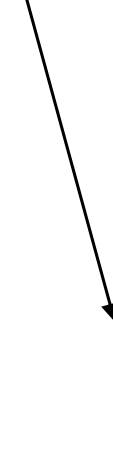

Full-text articles excluded, with reasons

( $n=118$ )

- Not primary article $(n=4)$

- Not peer reviewed $(n=73)$

- Language $(n=10)$

- No chest imaging discussed $(n=8)$

- No specific imaging features

discussed $(n=12)$

- COPD or exacerbation not

confirmed $(n=7)$

- Unable to extract data for COPD patients from other conditions $(n=$ 4)

Figure I PRISMA flow chart. Studies were excluded both at screening and at full-text review, leading to a total of $5 \mathrm{I}$ studies included in the qualitative synthesis.

discussion. After screening, the included studies were exported to another EndNote library and classified by imaging modality. The articles were then read in full and articles that were found to not meet inclusion criteria were excluded.

\section{Data Extraction}

A standardized data extraction table for capturing key information was developed before reading the full studies (Table 1). We also created a list of Imaging Biomarkers, included as Table S1. 
In addition to the imaging biomarkers, further relevant information was captured from each article. Where described in the articles, we recorded COPD severity when stable: depending on the availability of this information, preference was given to GOLD stage, followed by forced expiratory volume in 1 second $\left(\mathrm{FEV}_{1}\right) \%$ predicted and finally $\mathrm{FEV}_{1} / \mathrm{FVC}$ (forced vital capacity). We recorded the timing of imaging to describe when imaging was performed in relation to the exacerbation time-course. Information on ascertainment of clinical diagnosis of exacerbation was collected and used for quality assessment (see below).

\section{Quality Assessment}

Each study was assessed for quality using a 5-point grading system. We selected the Modified Newcastle-Ottawa Quality Assessment Scale (NOS) for Cohort Studies. ${ }^{8}$ It awards points to studies in three categories: Patient Selection (maximum 4 points), Comparability (maximum 2 points) and Outcome (maximum 3 points), allowing a maximum of 9 points for the highest quality studies. We modified the NOS to make it more applicable for the types of studies we were reporting - the original wording of each criterion from the NOS Scale, our modified decision rules in the context of imaging for COPD exacerbations and the maximum points awarded are described in Table S2. A confirmed clinical diagnosis of exacerbation was an inclusion criterion for our study, and the thoroughness of describing the criteria for confirming exacerbation contributed a point on our quality scale. For the modified criteria, the maximum score was 5 with a higher score representing higher quality. When synthesising evidence, we gave greatest weight to the studies of highest quality.

\section{Results}

\section{Search Results}

The search returned a total of 6,718 results. After removal of duplicates $(n=1,672)$, the final number of articles was 5,046 . One additional study was identified soon after publication by speaking to a clinician involved in its design.

After screening the titles and abstracts of the 5,047 articles, we excluded 4,878 for reasons outlined in the PRISMA Flowchart (Figure 1). ${ }^{3}$ We excluded 118 further articles at full-text assessment, for the reasons also outlined in Figure 1. Fifty-one studies proceeded to full data extraction and qualitative synthesis.

\section{Imaging Biomarkers of Exacerbations of COPD}

\section{Included Studies}

Table 1 presents the findings of the 51 studies included in the final analysis. In general, most studies discussed imaging biomarkers either as prevalence values of radiological findings in a population of subjects with an exacerbation of COPD, or as quantitative measurements of an imaging feature. A total of 10 of the 51 papers $^{9-18}$ presented quantitative measurements of imaging features at exacerbation. One paper presented quantitative individual patient data, but no cohort averages, ${ }^{19}$ and the remaining 40 papers presented prevalence values for established radiological or other features. Most studies that presented quantitative values for imaging biomarkers performed consecutive imaging for the same subjects (at exacerbation compared to the same subjects at stable state as controls), whilst one study ${ }^{13}$ compared exacerbating subjects with a group of different stable-state COPD controls.

The identified imaging features are summarised in Table S1. Ten papers ${ }^{20-29}$ focused only on the prevalence of pulmonary embolism (PE) in patients hospitalized for suspected exacerbation of COPD. Most of these did not include details of any other imaging features and so are useful only in estimating the frequency of PE at exacerbation. Eleven serial imaging studies ${ }^{10-12,14-19,30,31}$ performed imaging at multiple time-points - usually at exacerbation and subsequent follow-up (assumed to represent recovery). The follow-up period for the second imaging time-point ranged from several days ${ }^{17}$ to 16 months after the initial exacerbation imaging. ${ }^{16}$ In all the studies, the end of exacerbation was clinically confirmed.

There were two studies which performed imaging both before and after exacerbation: Wells et $\mathrm{al}^{15}$ investigated changes in the pulmonary artery and aorta on CT, whilst Kirby et $\mathrm{al}^{16}$ studied ventilation and diffusion within the lung using hyperpolarized helium MRI, but only in a single subject.

A total of 36 imaging features present during an exacerbation were reported by the included studies (Table S1). Conclusions, summarized in Figure 2, were primarily drawn from the 10 studies $^{10-15,17-19,30}$ where a control group at stable state was present and the study scored three or more points on the Quality Assessment scale.

\section{Chest CT}

We identified 19 studies that reported chest computed tomography (CT) scanning during an exacerbation 


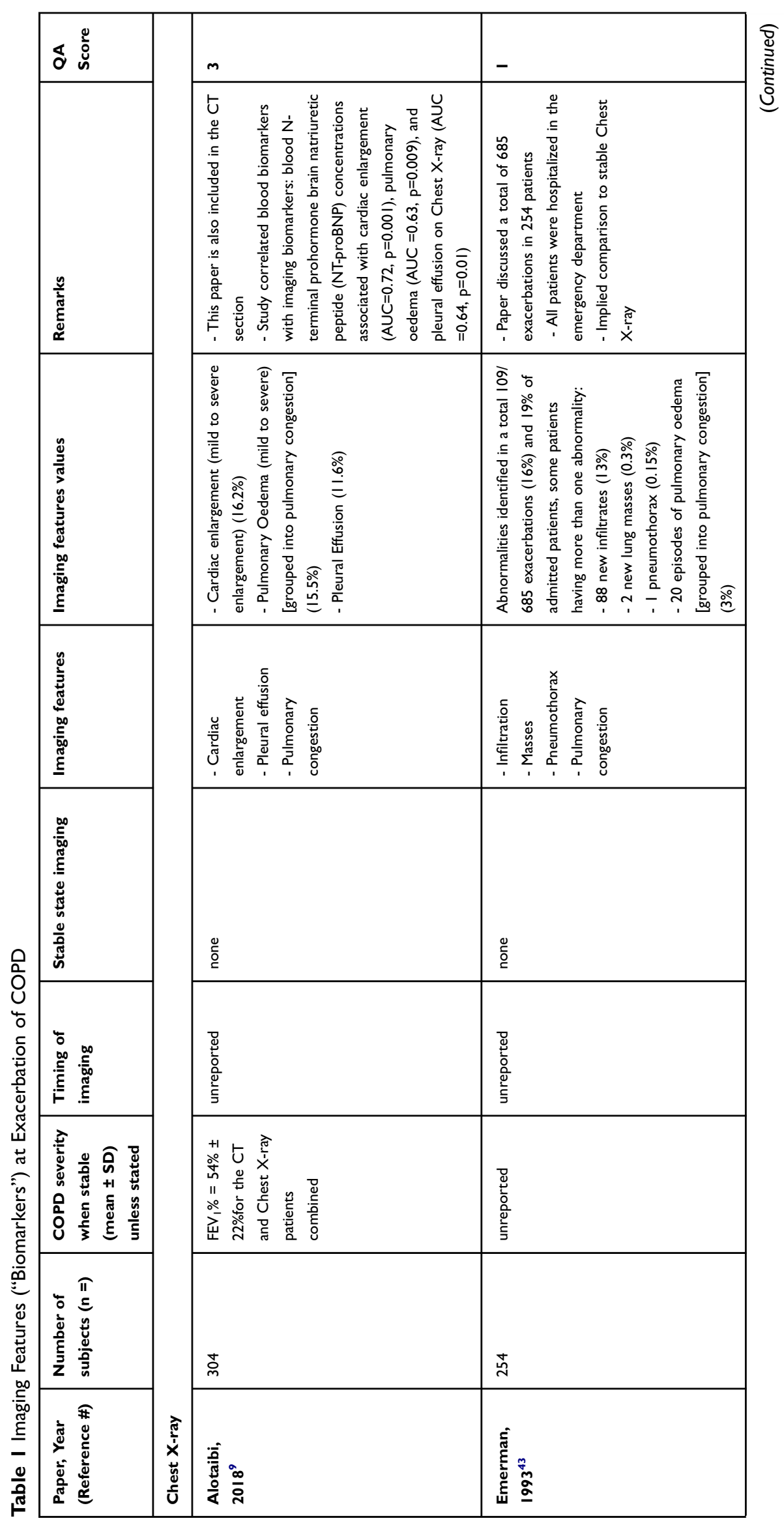




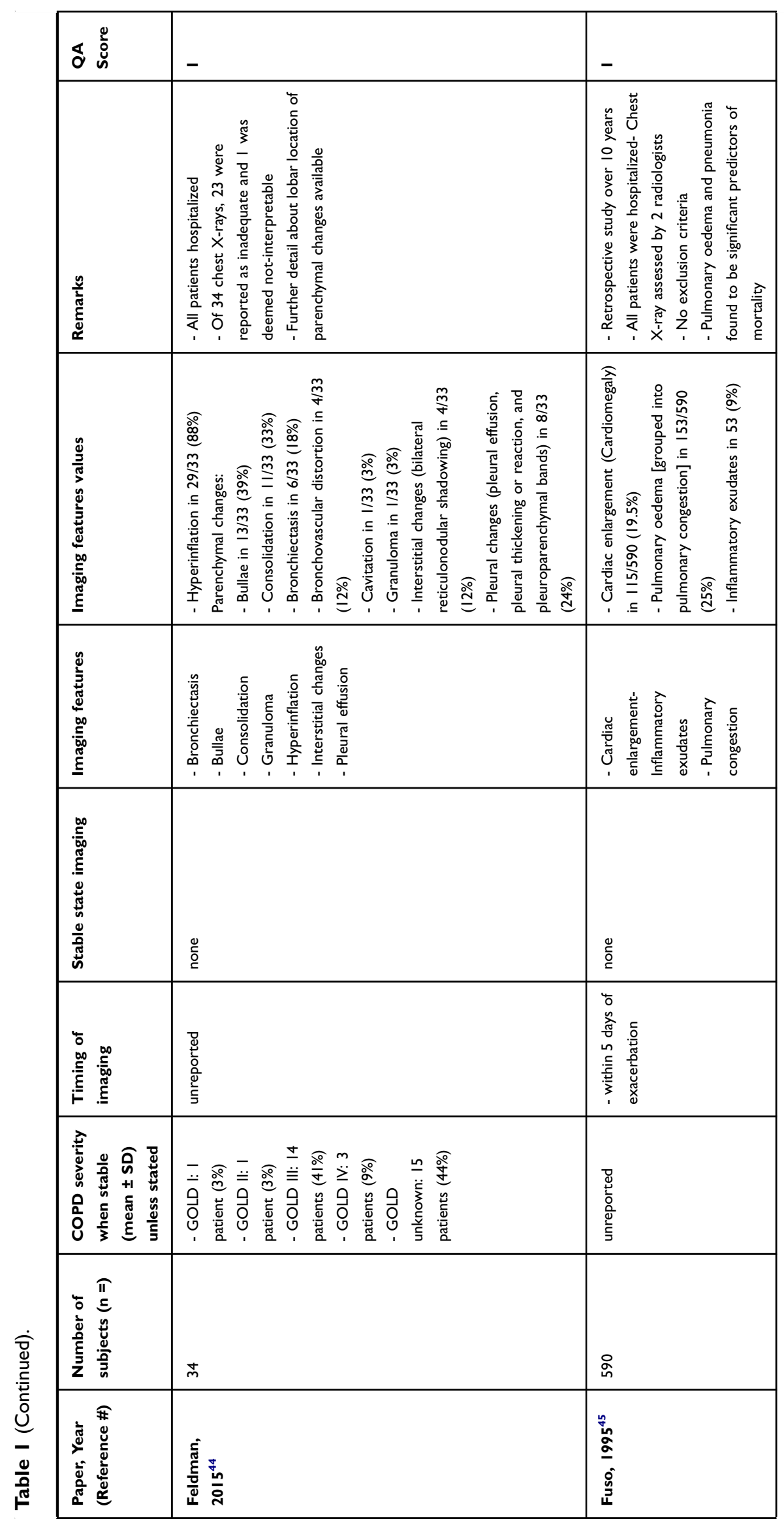




\begin{tabular}{|c|c|}
\hline N & $N$ \\
\hline 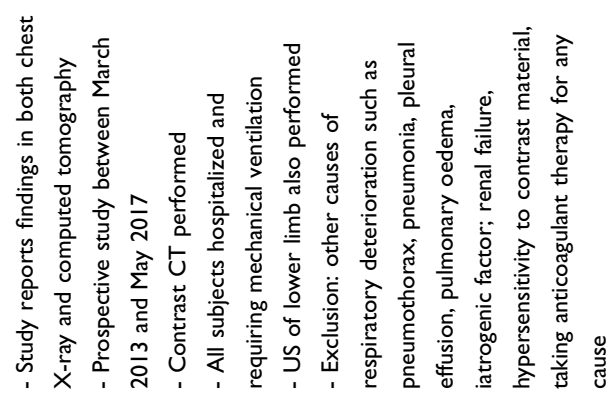 & 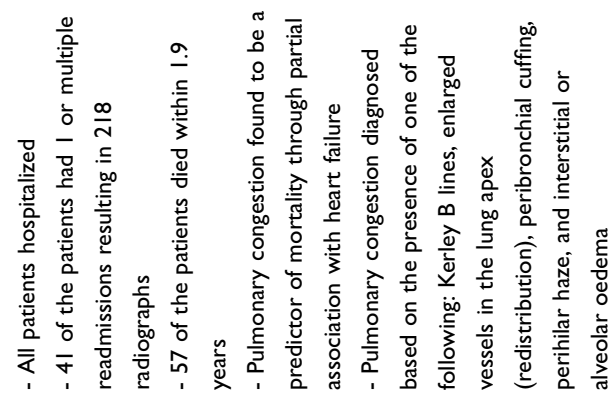 \\
\hline 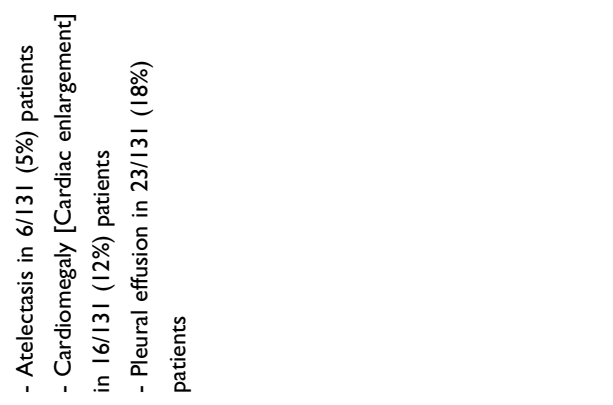 & 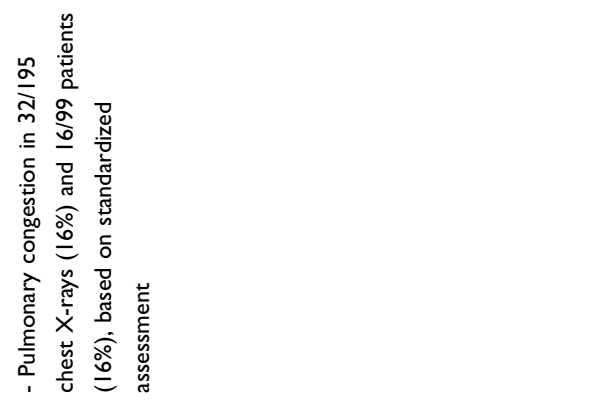 \\
\hline 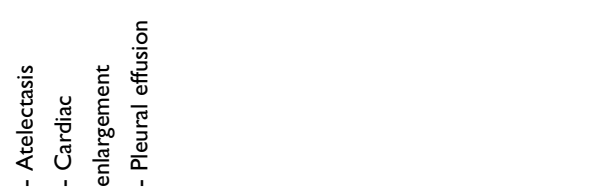 & 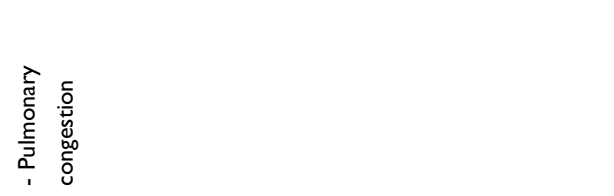 \\
\hline 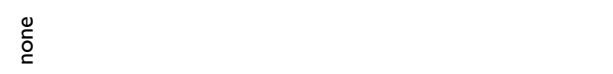 & $\begin{array}{l}\stackrel{0}{0} \\
\check{c}\end{array}$ \\
\hline 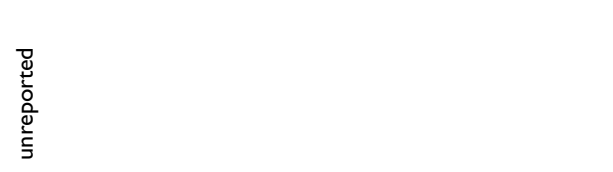 & 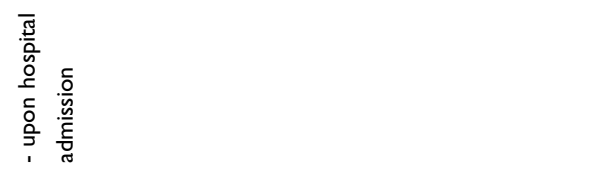 \\
\hline 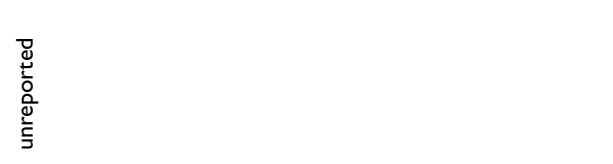 & 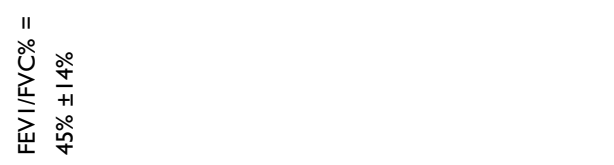 \\
\hline$\overline{\underline{m}}$ & $\sigma$ \\
\hline 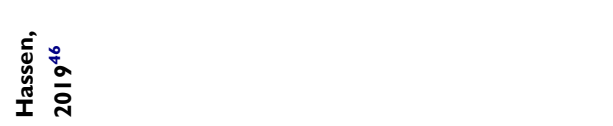 & 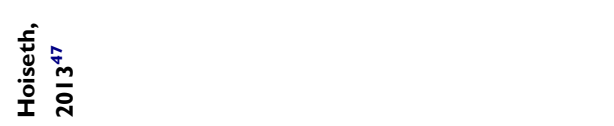 \\
\hline
\end{tabular}




\begin{tabular}{|c|c|c|}
\hline$\triangleleft \stackrel{0}{\circ}$ & $m$ & - \\
\hline 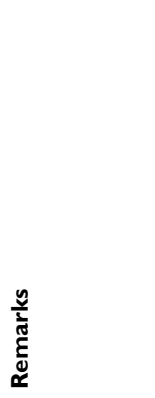 & 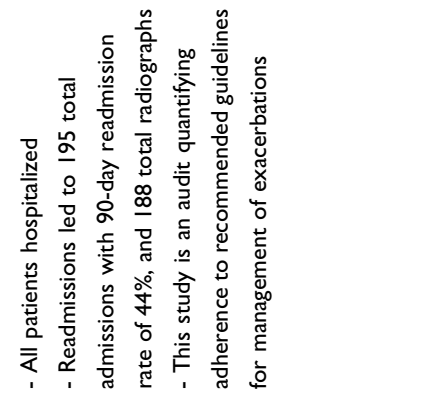 & 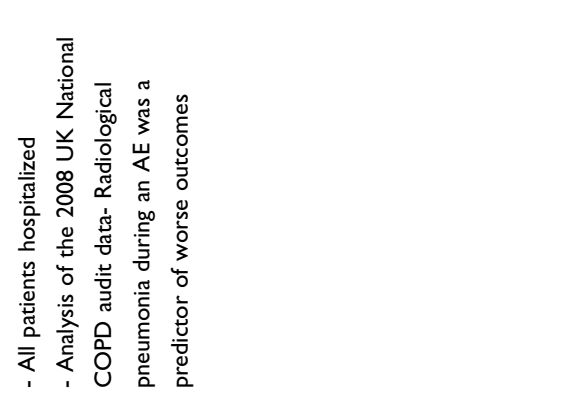 \\
\hline 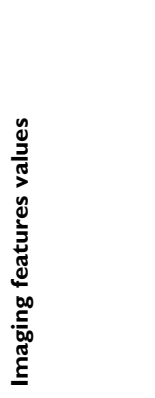 & 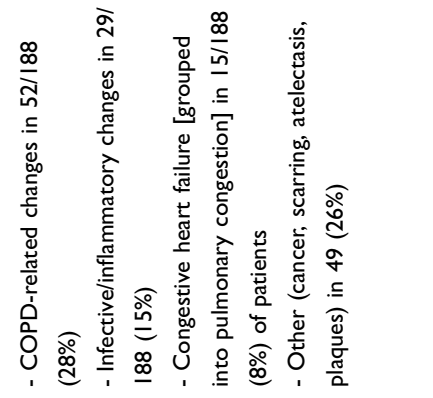 & 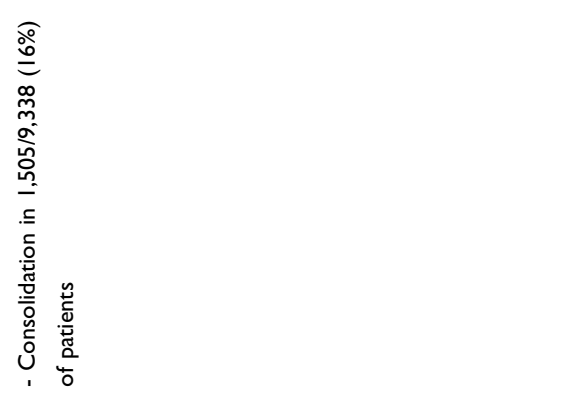 \\
\hline 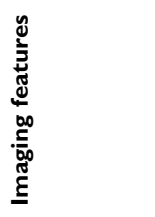 & 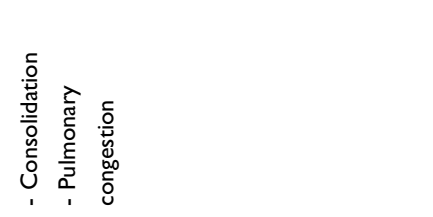 & 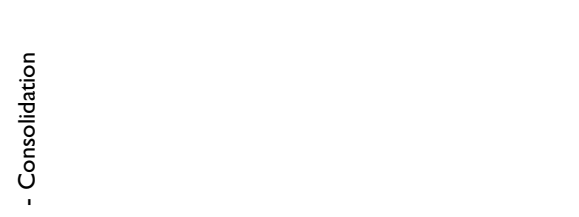 \\
\hline 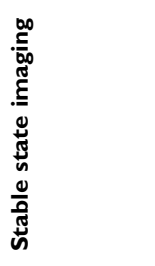 & 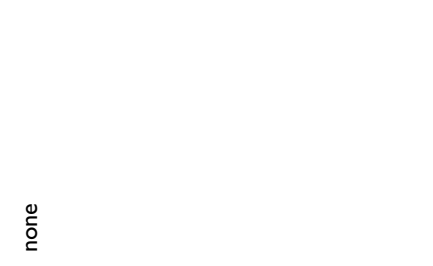 & $\begin{array}{l}0 \\
\stackrel{0}{0}\end{array}$ \\
\hline 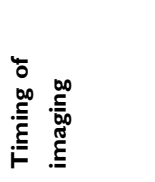 & 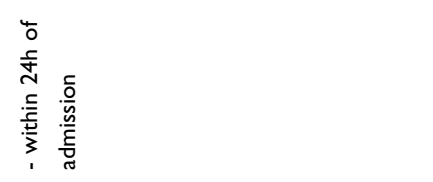 & 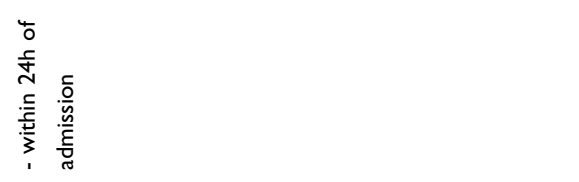 \\
\hline 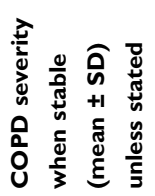 & 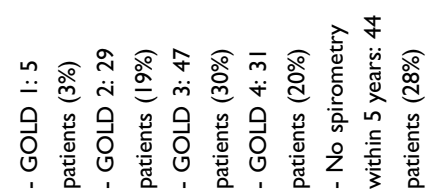 & 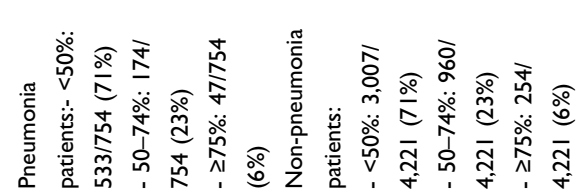 \\
\hline 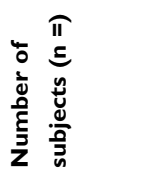 & $\stackrel{\stackrel{\circ}{~}}{ }$ & $\underset{\substack{m \\
\sigma}}{\sigma^{2}}$ \\
\hline 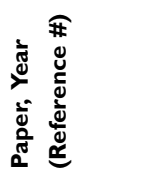 & 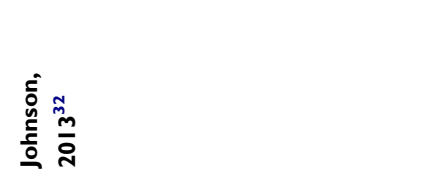 & 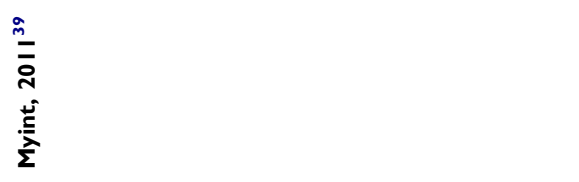 \\
\hline
\end{tabular}




\begin{tabular}{|c|c|c|}
\hline$m$ & $N$ & $m$ \\
\hline 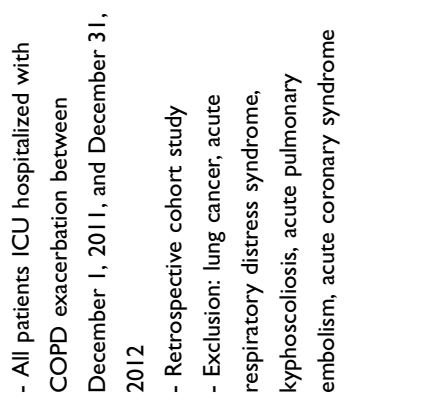 & 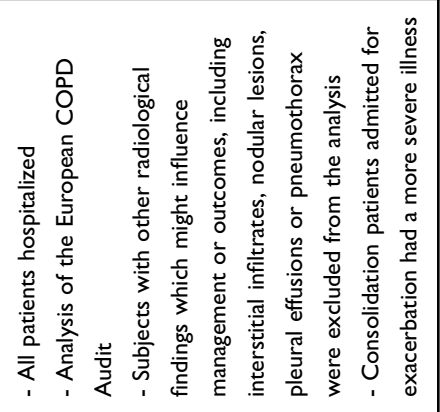 & 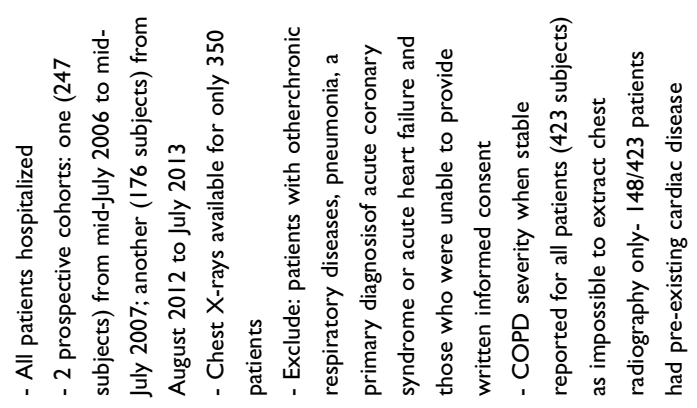 \\
\hline 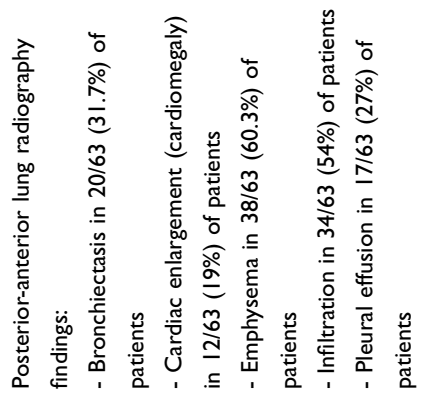 & 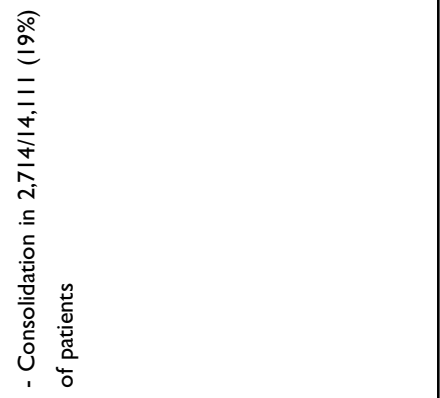 & 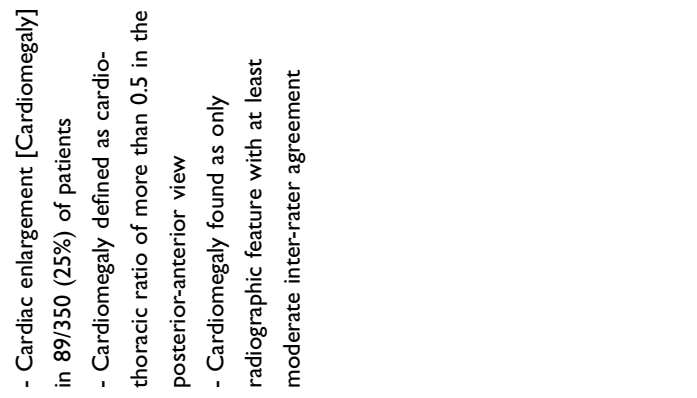 \\
\hline 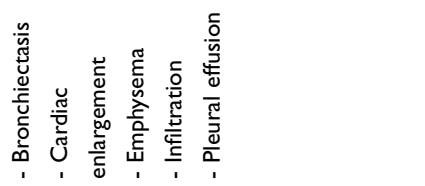 & 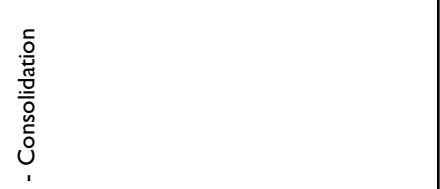 & 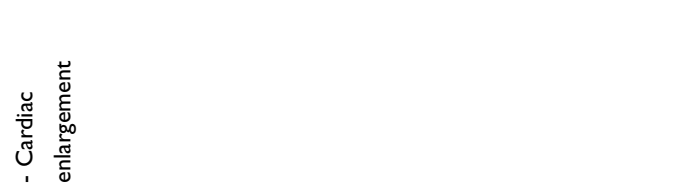 \\
\hline $\begin{array}{l}\stackrel{0}{0} \\
\text { zo }\end{array}$ & $\stackrel{\circ}{\check{\sigma}}$ & 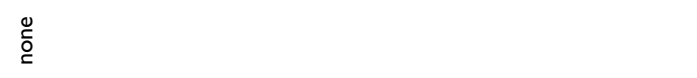 \\
\hline 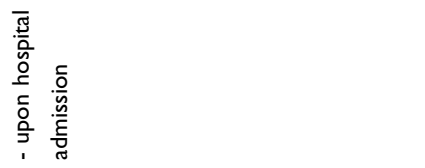 & 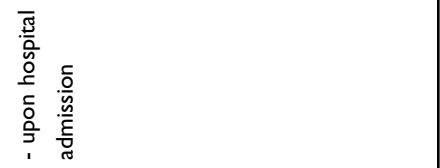 & 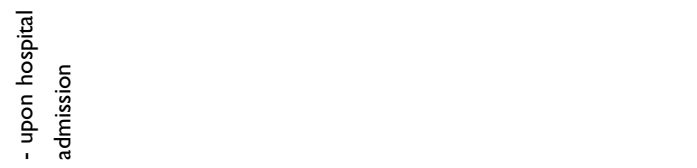 \\
\hline 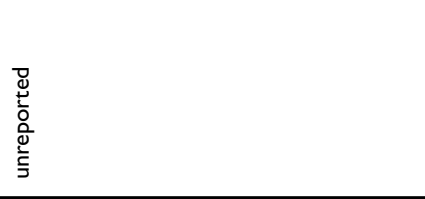 & 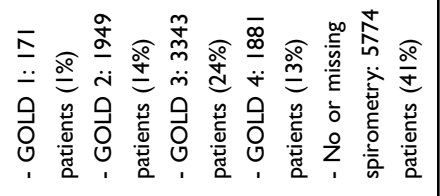 & 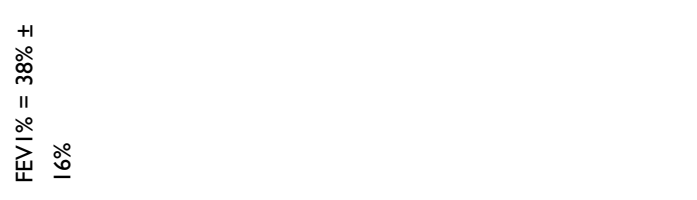 \\
\hline กิ & $\overline{\bar{\Xi}}$ & 品 \\
\hline 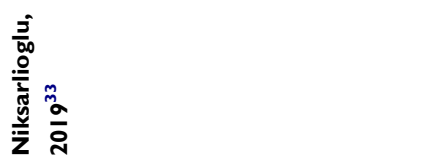 & 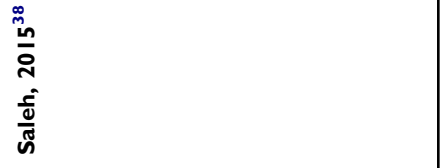 & 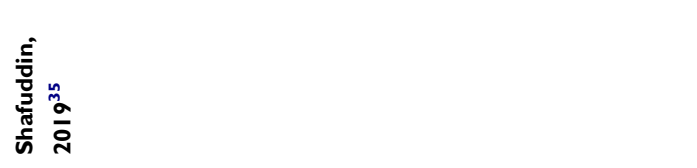 \\
\hline
\end{tabular}




\begin{tabular}{|c|c|c|c|}
\hline ઠ & N & $N$ & $N$ \\
\hline 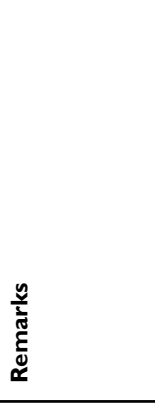 & 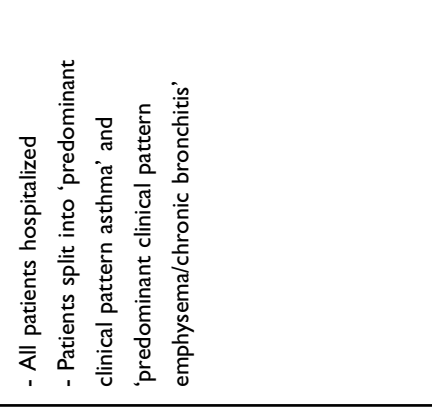 & 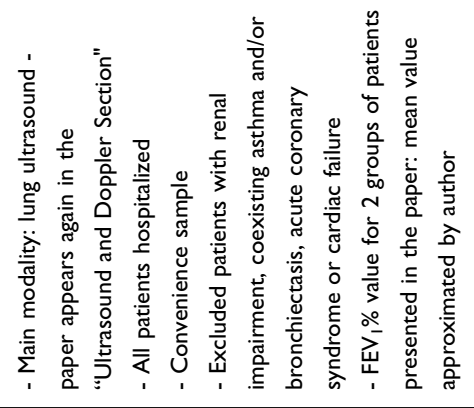 & 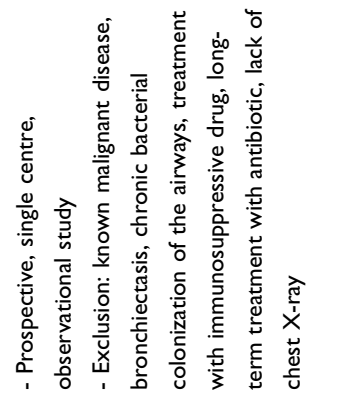 \\
\hline 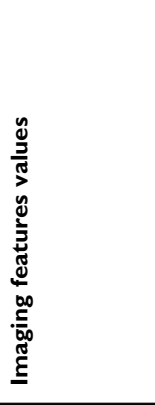 & 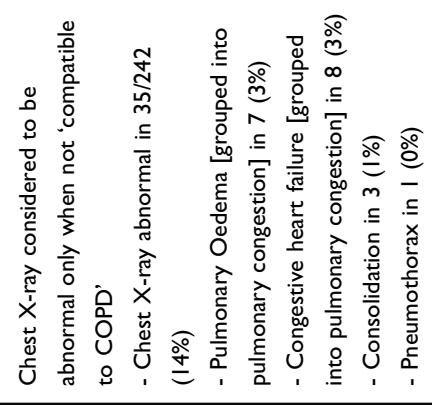 & 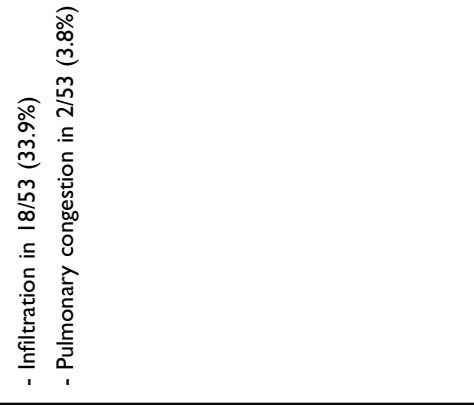 & 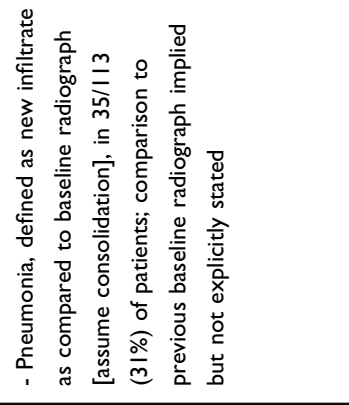 \\
\hline 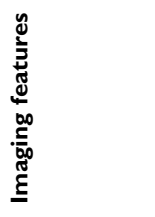 & 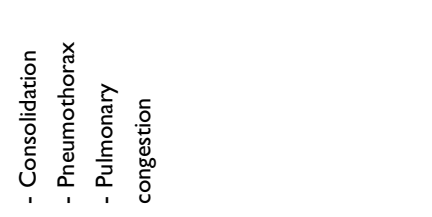 & 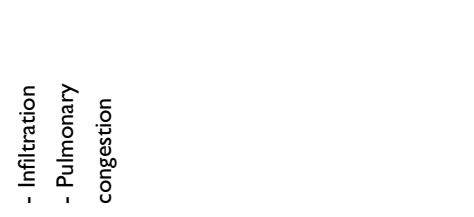 & 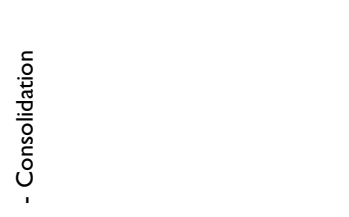 \\
\hline 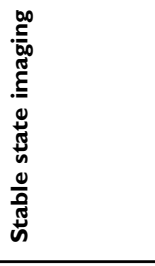 & $\begin{array}{l}0 \\
\stackrel{0}{\sigma} \\
\end{array}$ & $\begin{array}{l}0 \\
\stackrel{0}{0} \\
\end{array}$ & 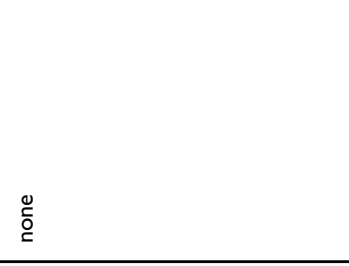 \\
\hline 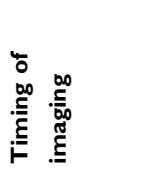 & 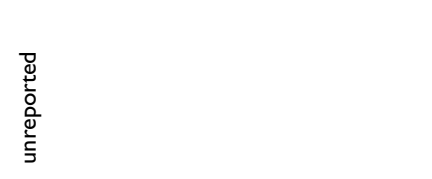 & 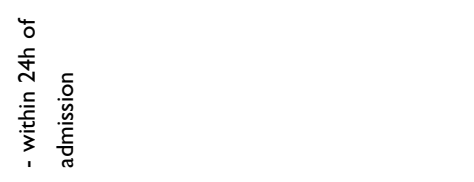 & 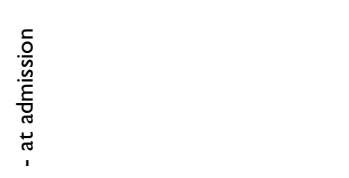 \\
\hline 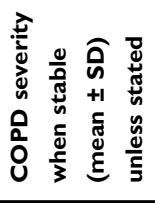 & 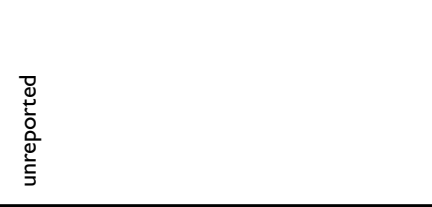 & 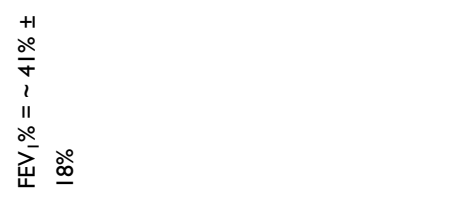 & 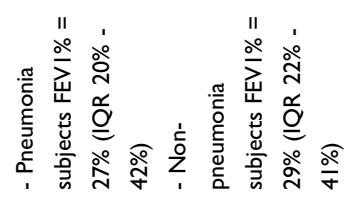 \\
\hline 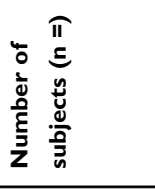 & $\stackrel{\mathcal{A}}{\mathcal{N}}$ & $\tilde{n}$ & $\stackrel{m}{=}$ \\
\hline 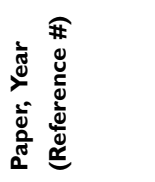 & 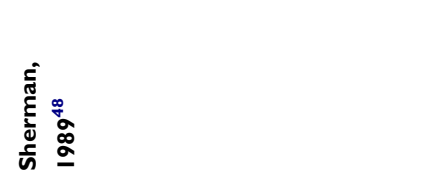 & 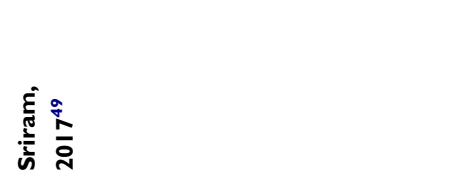 & 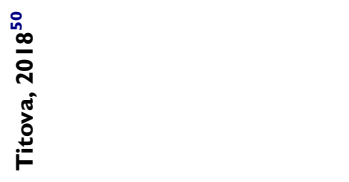 \\
\hline
\end{tabular}




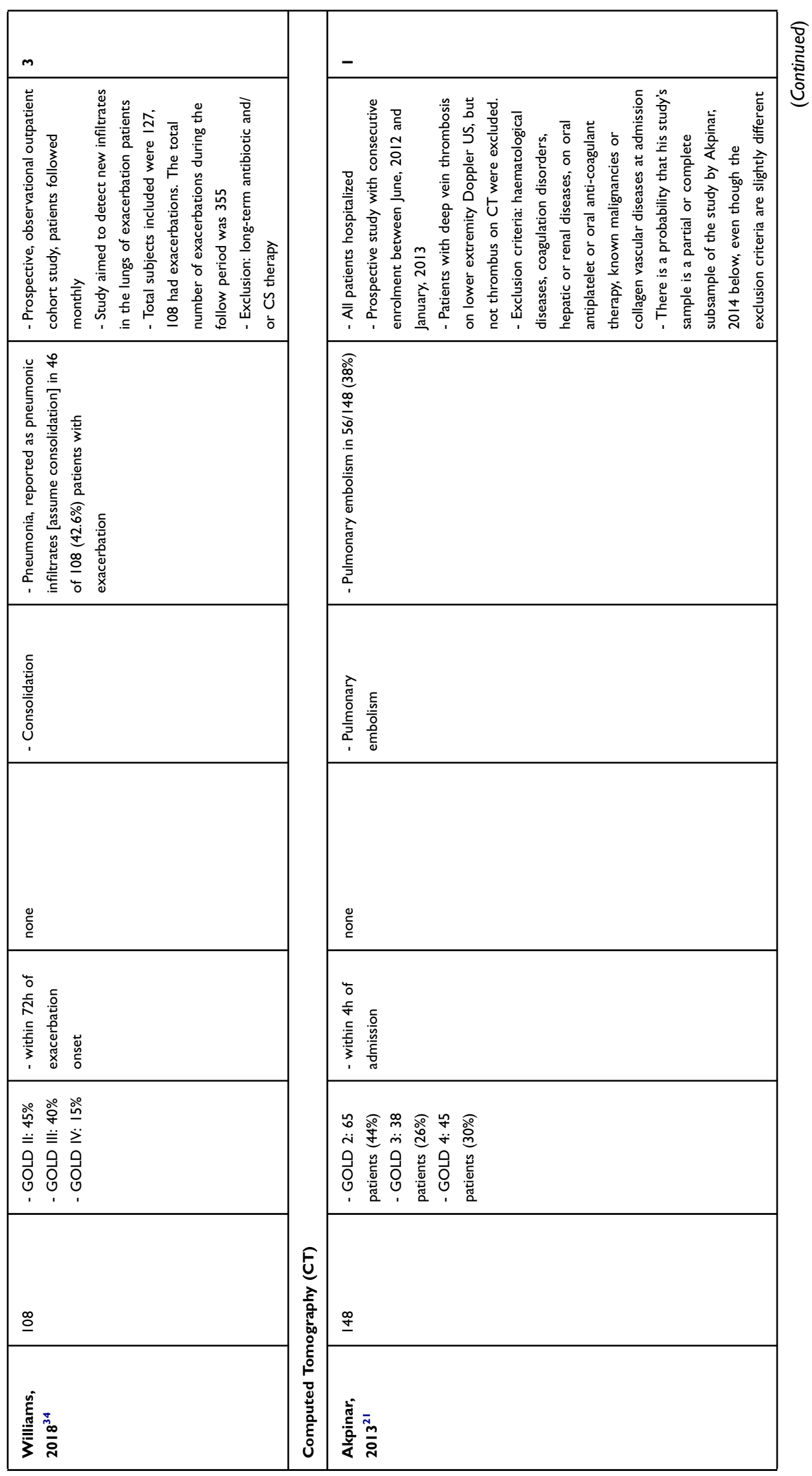




\begin{tabular}{|c|c|c|}
\hline هั & $m$ & $m$ \\
\hline 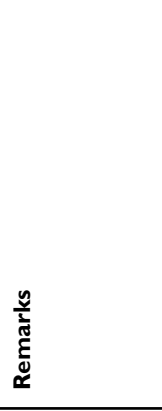 & 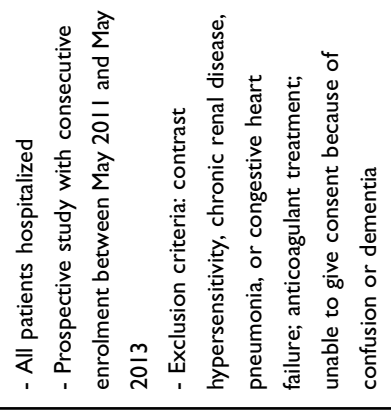 & 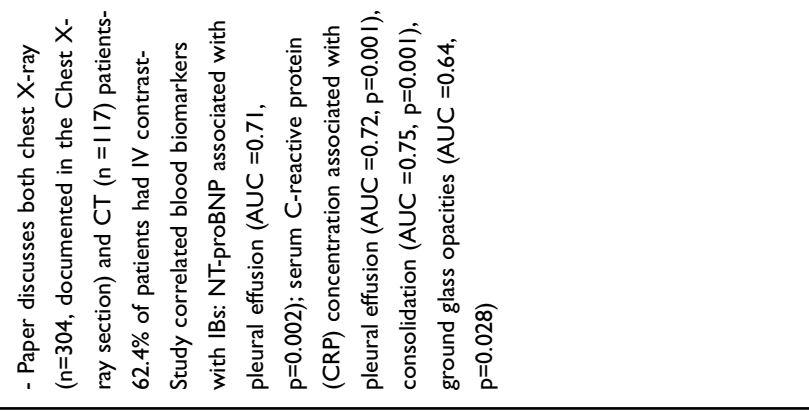 \\
\hline 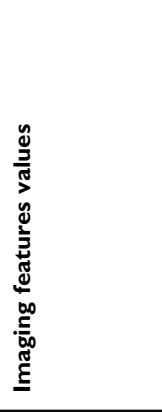 & 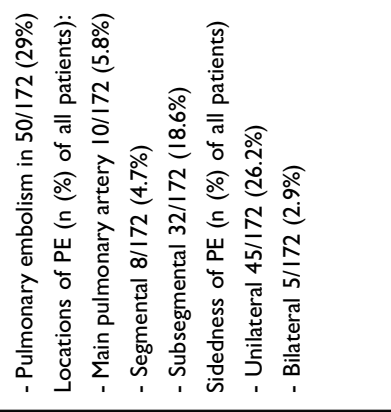 & 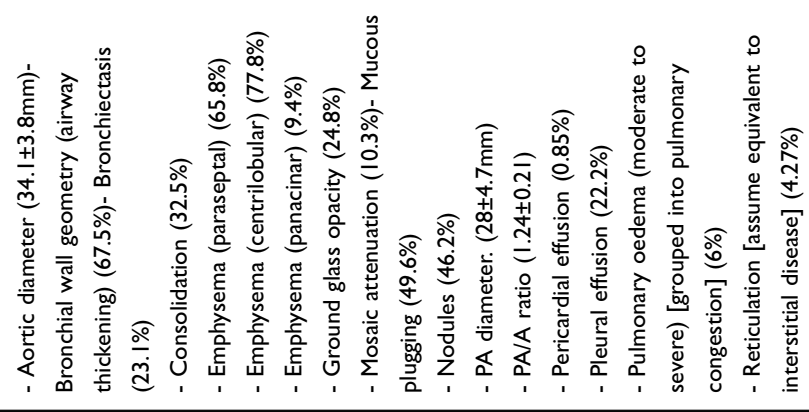 \\
\hline 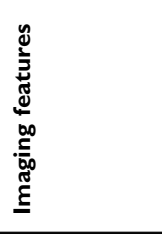 & 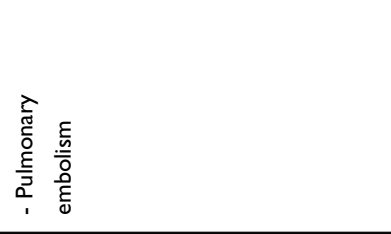 & 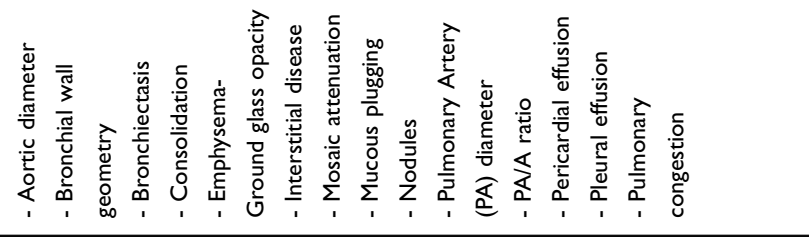 \\
\hline 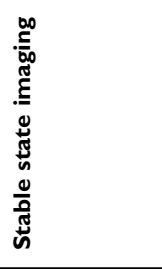 & 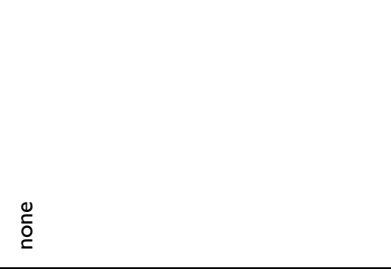 & 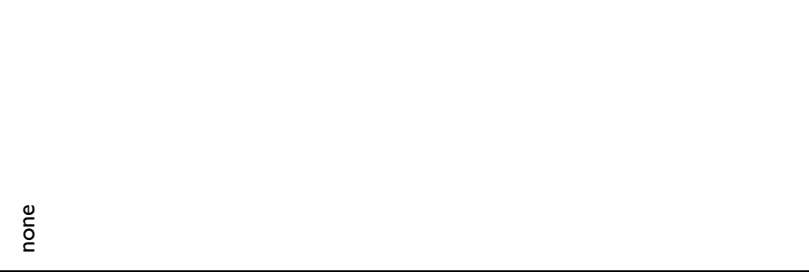 \\
\hline 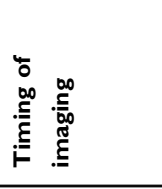 & 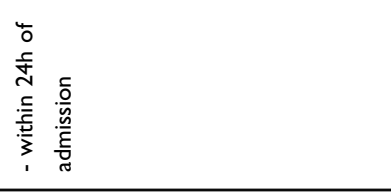 & 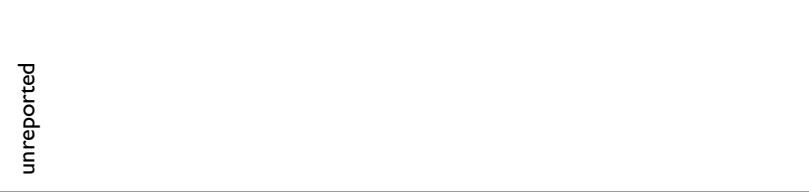 \\
\hline 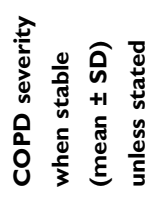 & 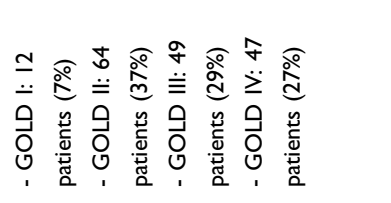 & 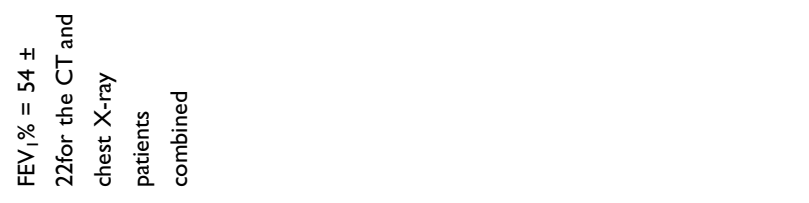 \\
\hline 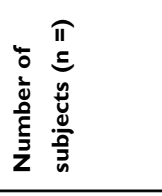 & $\underline{\underline{I}}$ & $\Xi$ \\
\hline 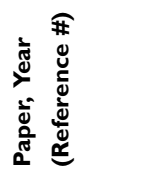 & 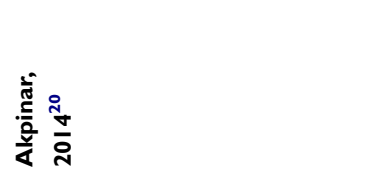 & 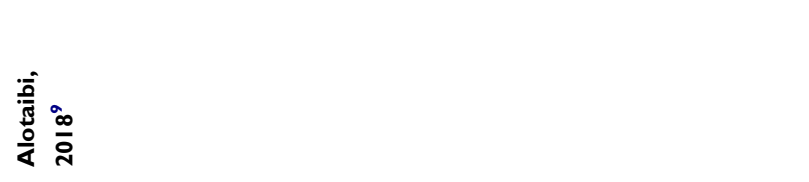 \\
\hline
\end{tabular}




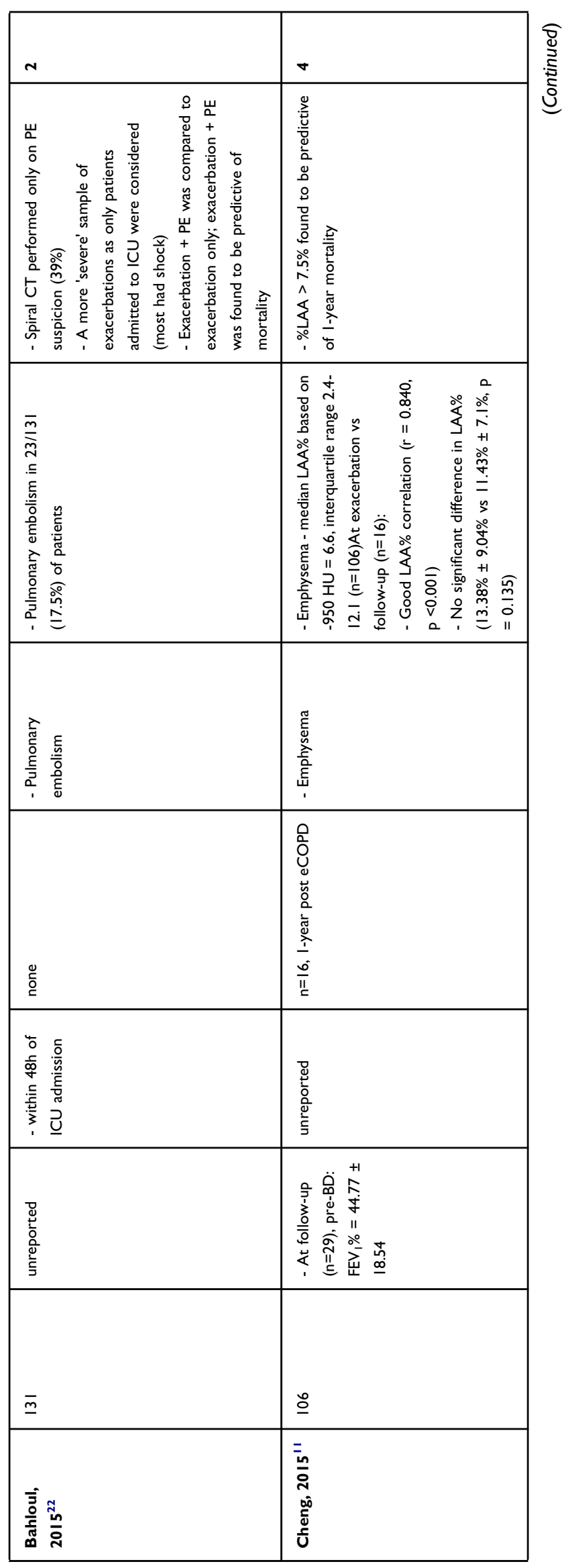




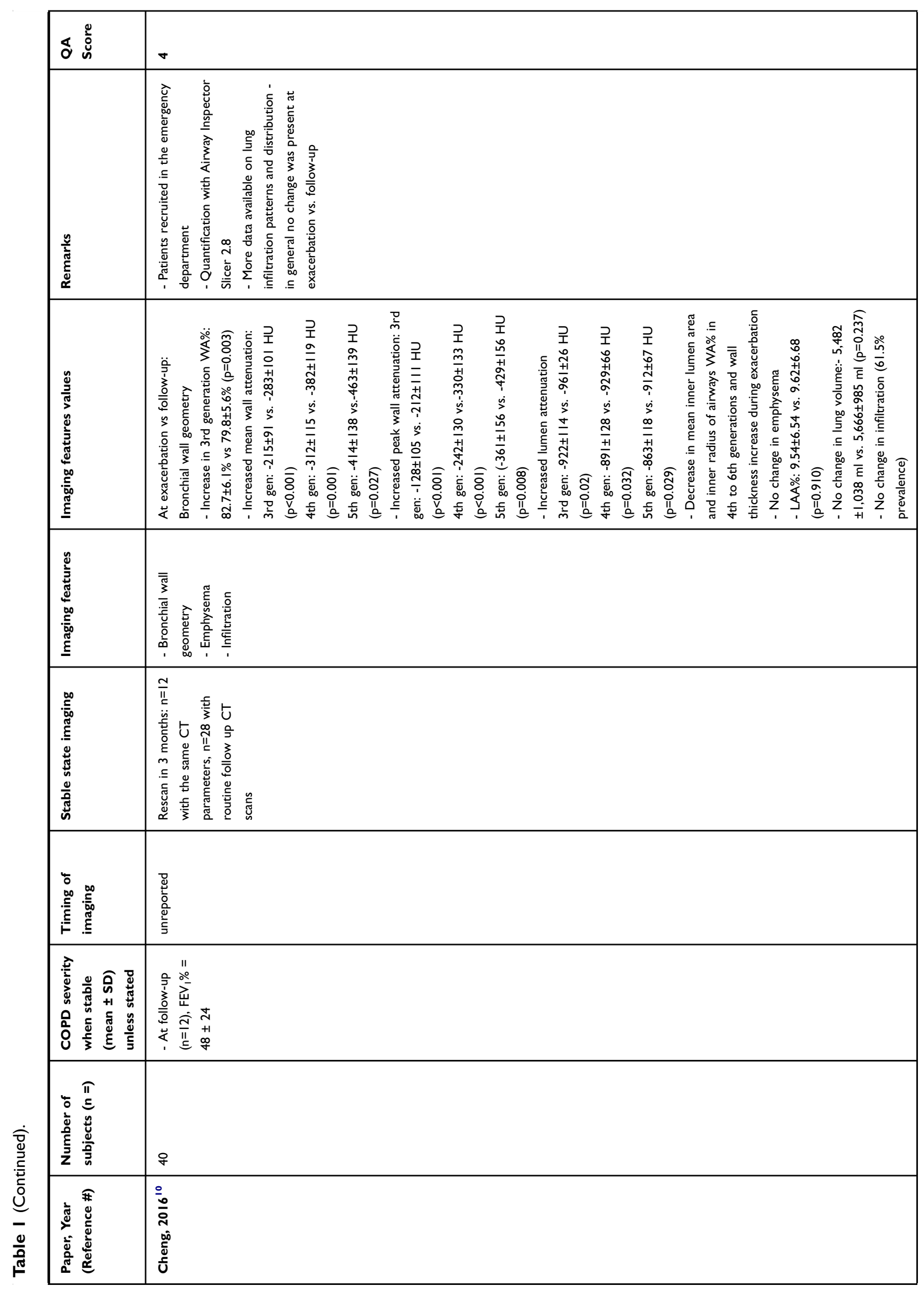




\begin{tabular}{|c|c|c|}
\hline N & $m$ & N \\
\hline 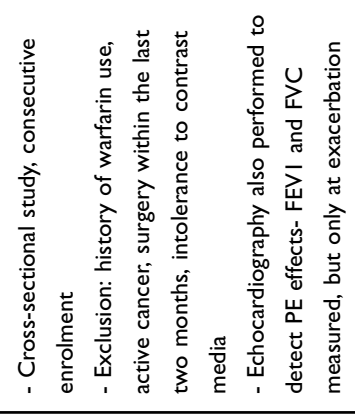 & 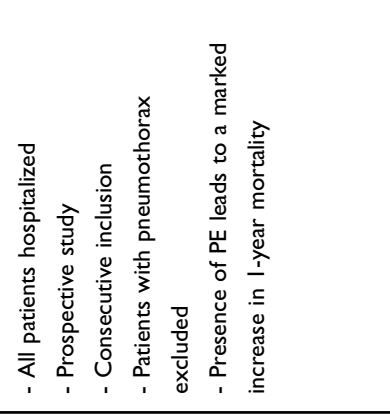 & 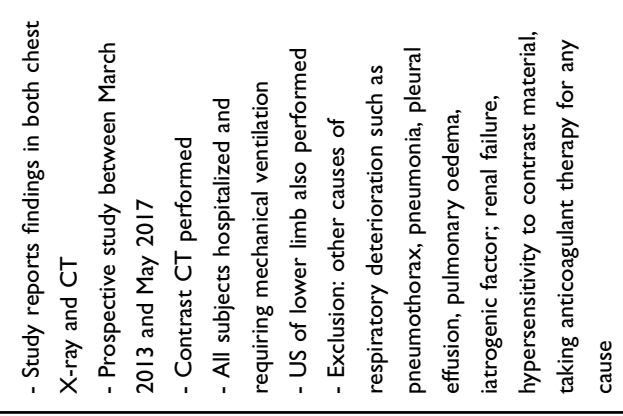 \\
\hline 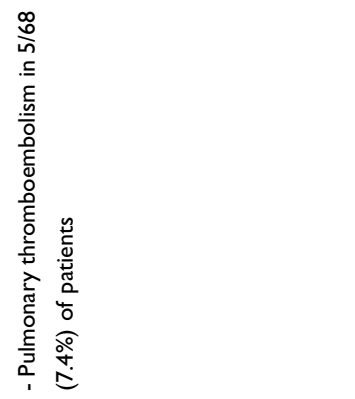 & 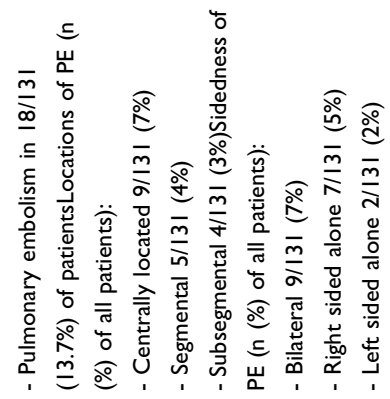 & 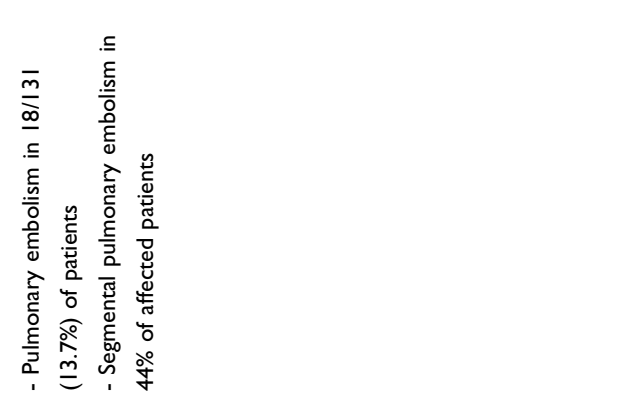 \\
\hline 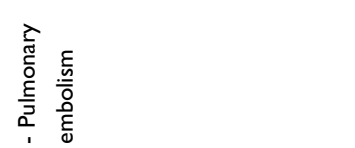 & 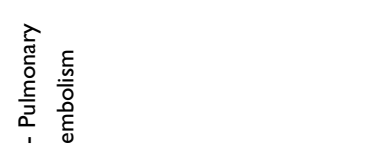 & 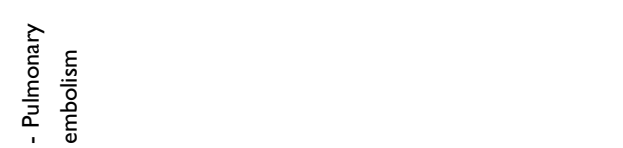 \\
\hline$\frac{\circ}{\stackrel{\circ}{\circ}}$ & 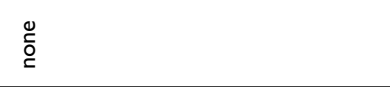 & 营 \\
\hline 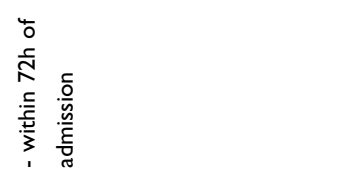 & 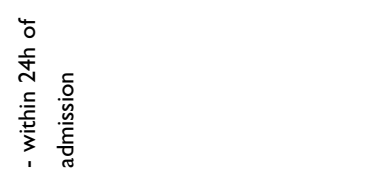 & 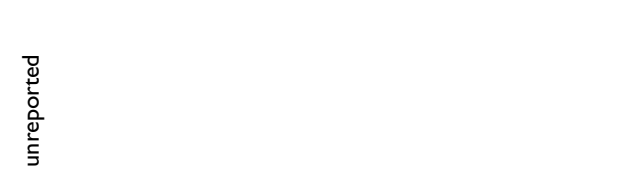 \\
\hline 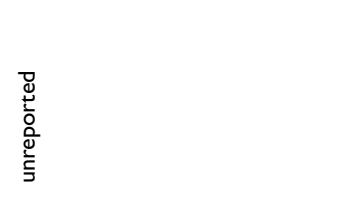 & 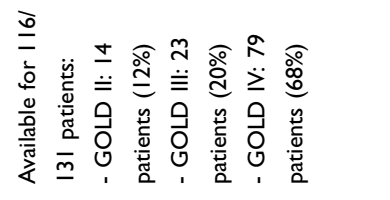 & 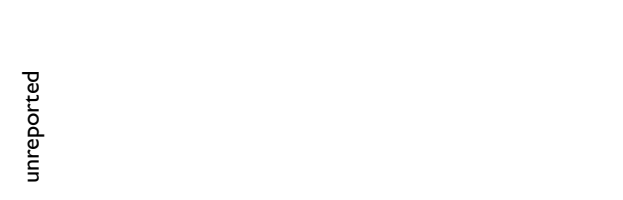 \\
\hline$\stackrel{\infty}{\infty}$ & $\overline{\underline{m}}$ & $\overline{\underline{m}}$ \\
\hline 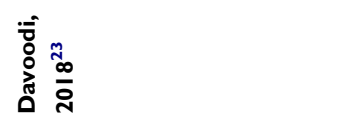 & 离 & 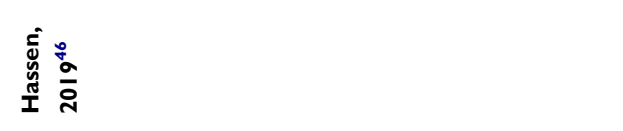 \\
\hline
\end{tabular}




\begin{tabular}{|c|c|c|c|}
\hline ১ & $\sigma$ & in & 0 \\
\hline 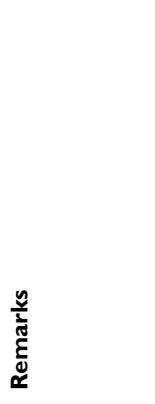 & 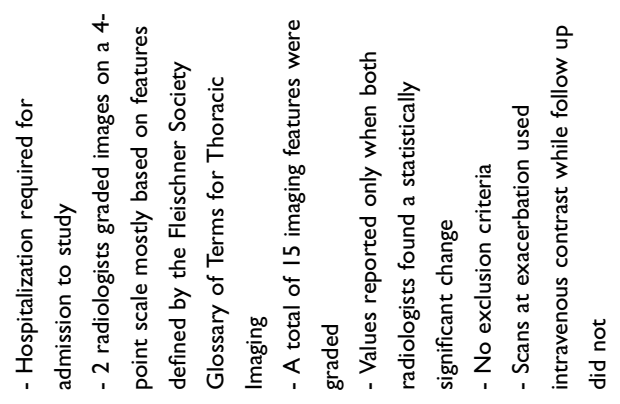 & 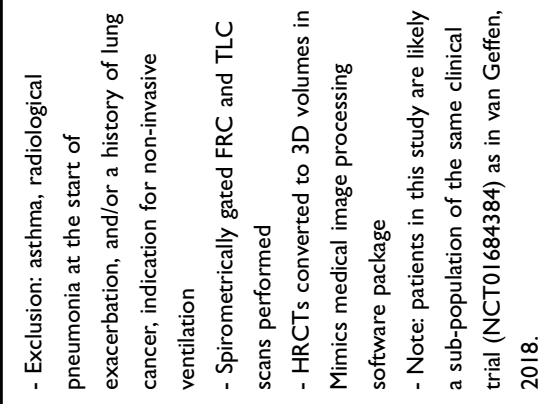 & 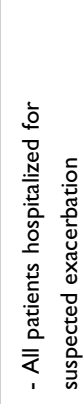 \\
\hline 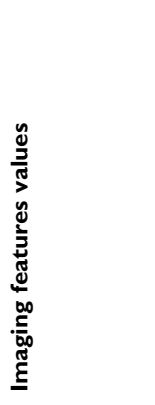 & 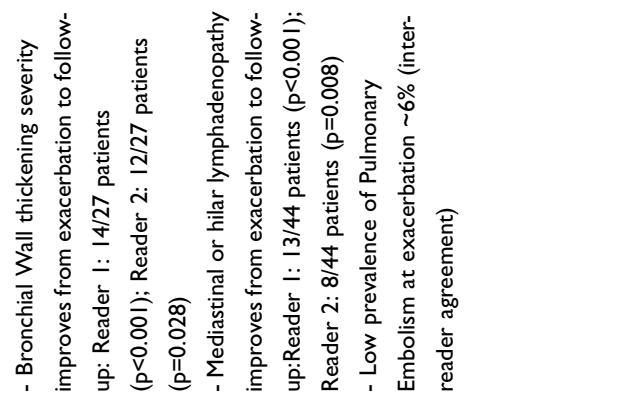 & 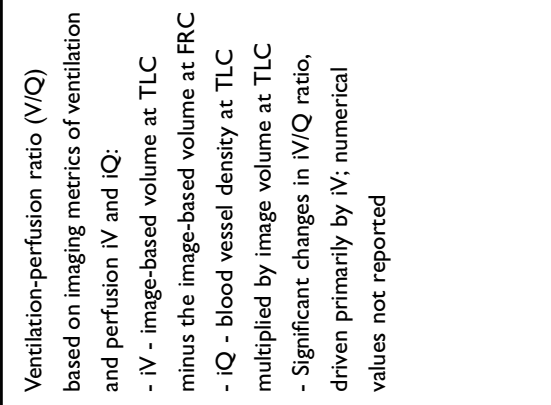 & 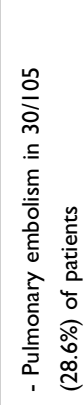 \\
\hline 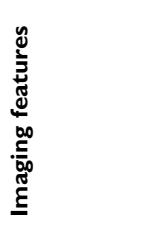 & 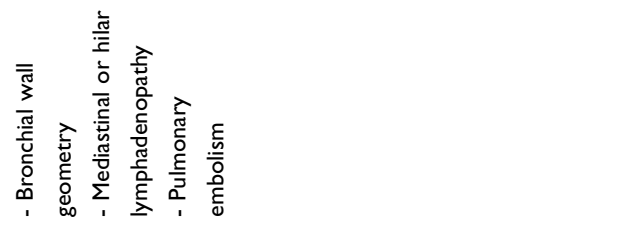 & 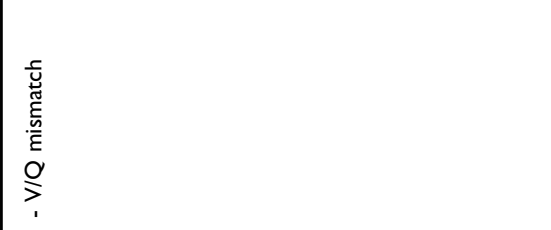 & 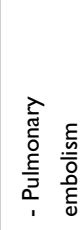 \\
\hline 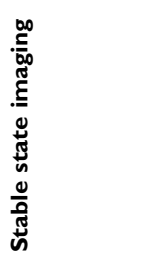 & 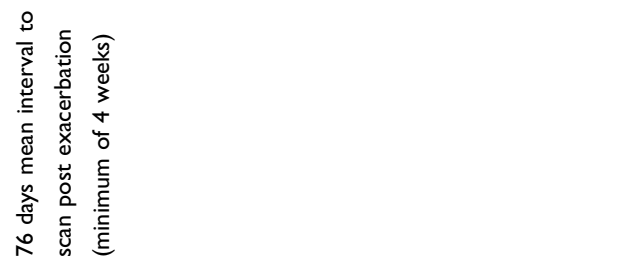 & 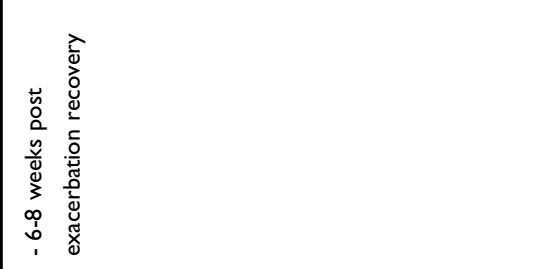 & $\begin{array}{l}0 \\
\stackrel{0}{0} \\
\end{array}$ \\
\hline 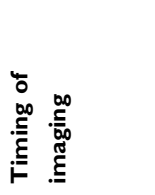 & & 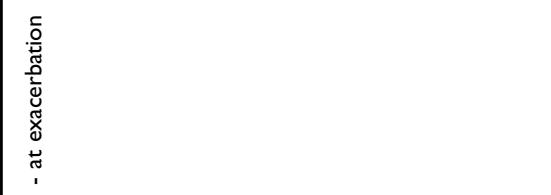 & 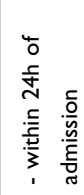 \\
\hline 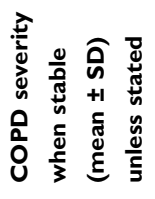 & 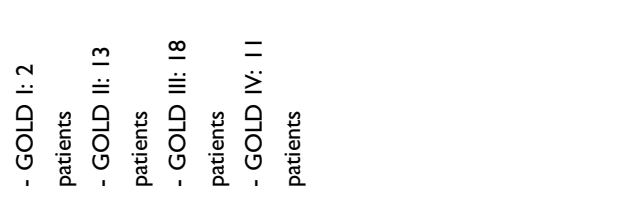 & 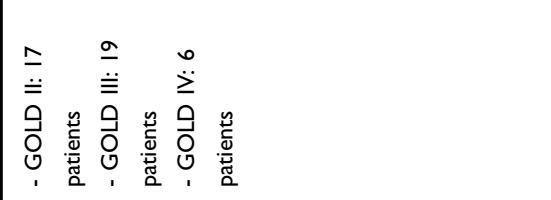 & 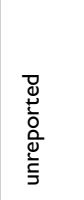 \\
\hline 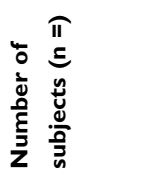 & ₹ & $\mathcal{F}$ & $\stackrel{ㅇ ㅡ}{1}$ \\
\hline 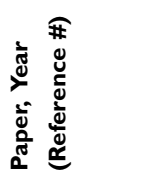 & 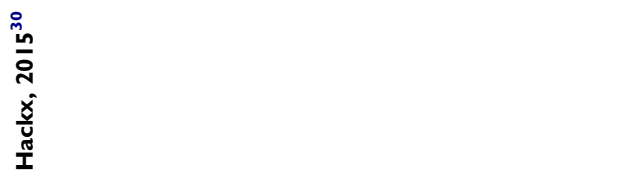 & 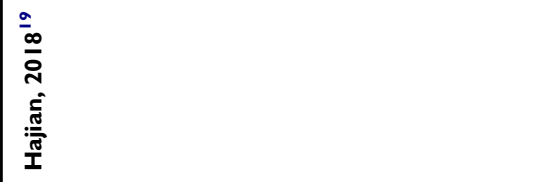 & 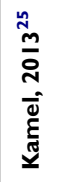 \\
\hline
\end{tabular}




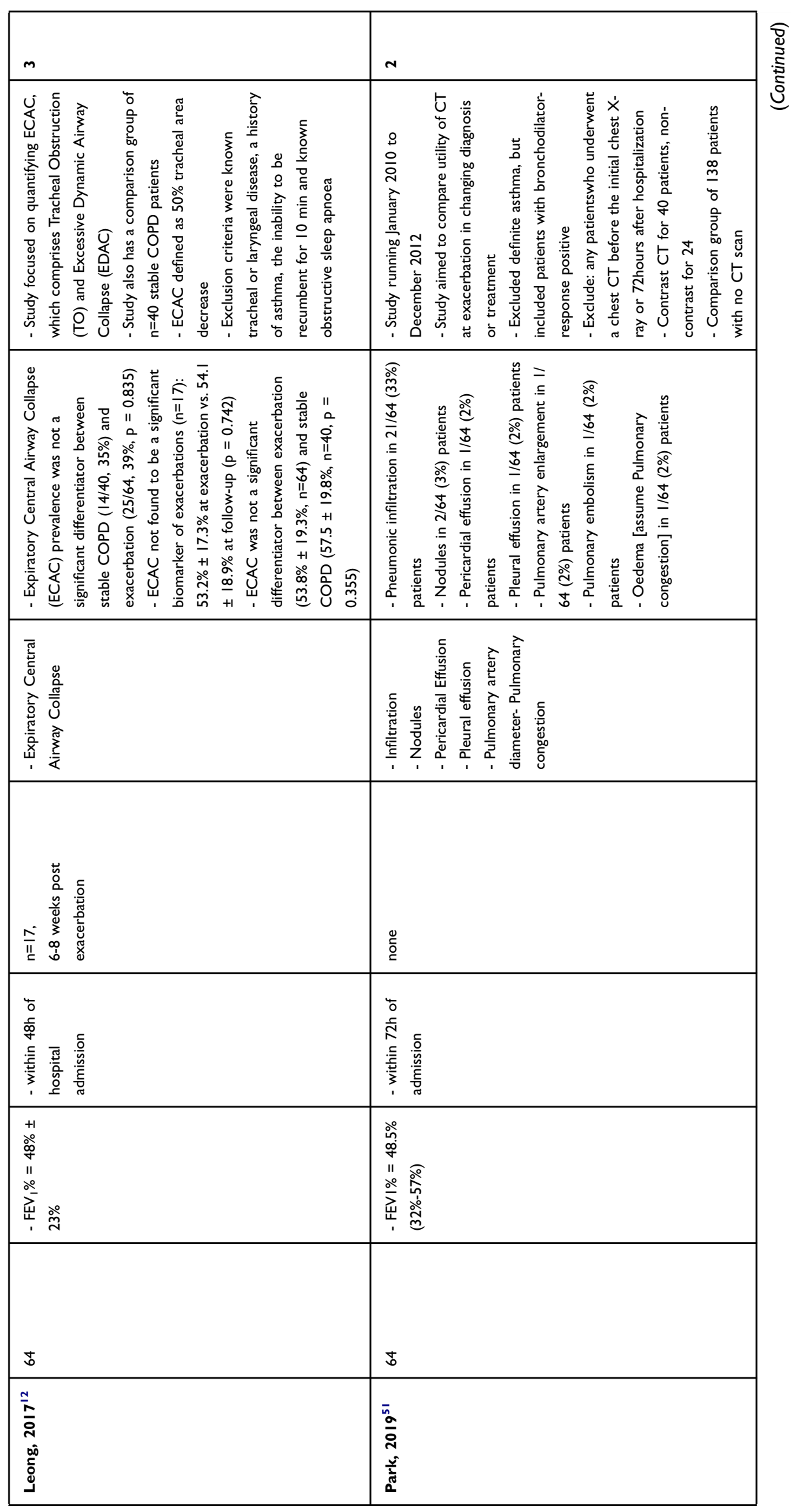




\begin{tabular}{|c|c|c|}
\hline む㐫 & $N$ & $N$ \\
\hline 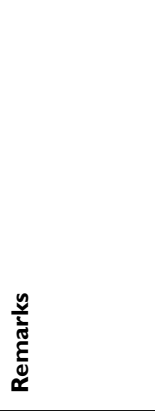 & 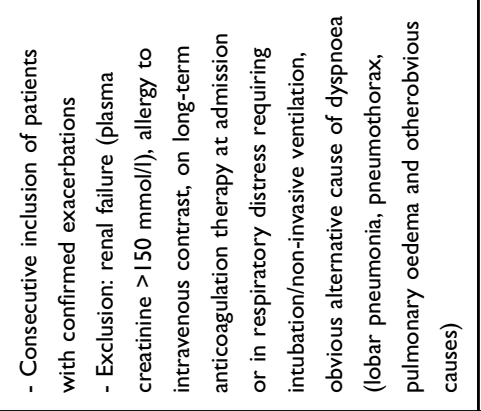 & 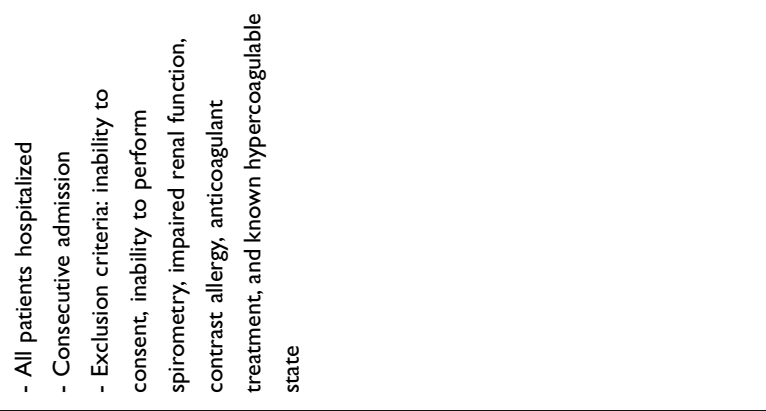 \\
\hline 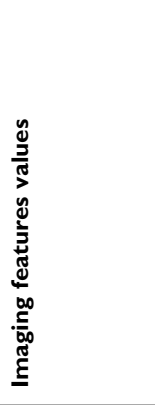 & 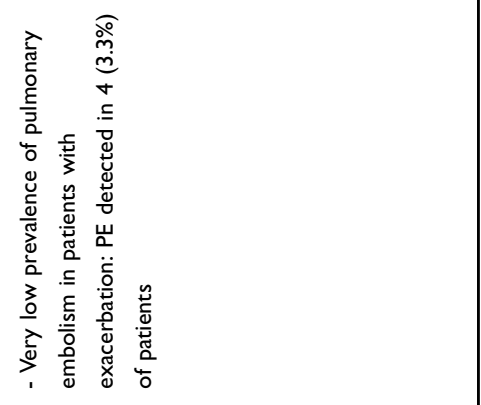 & 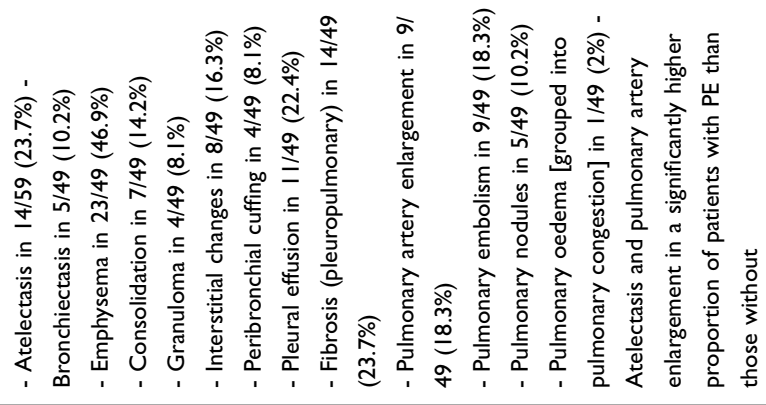 \\
\hline 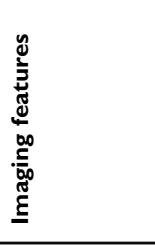 & 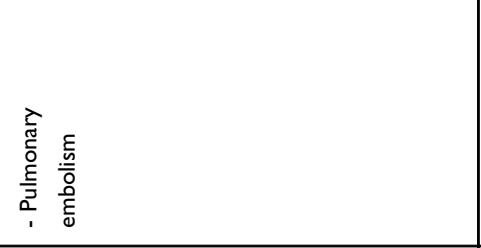 & 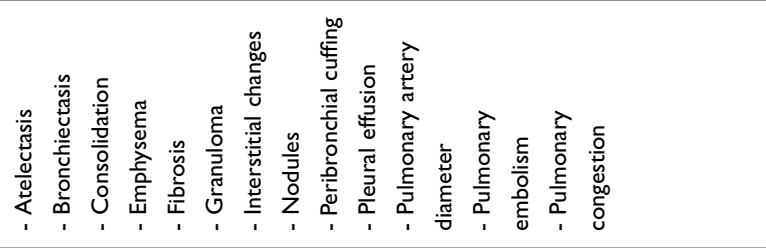 \\
\hline 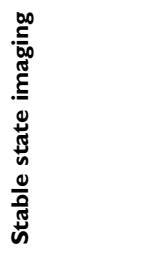 & $\begin{array}{l}\stackrel{0}{0} \\
\stackrel{0}{c}\end{array}$ & 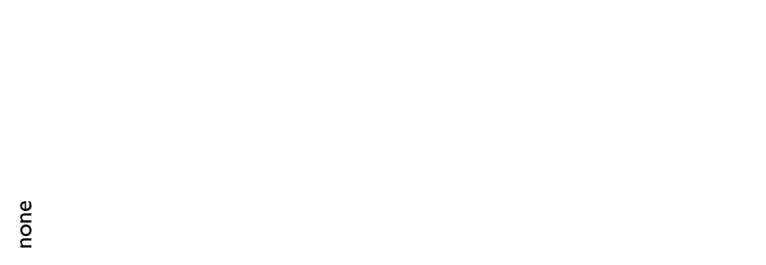 \\
\hline 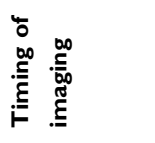 & 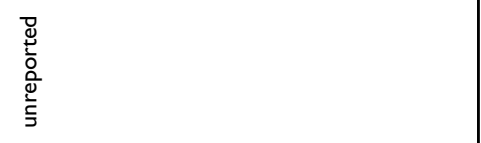 & 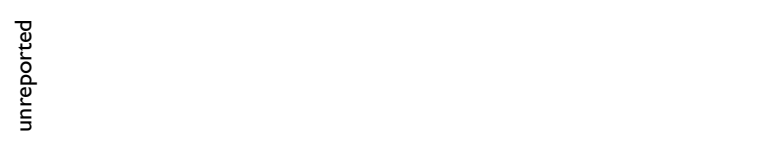 \\
\hline 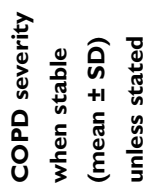 & 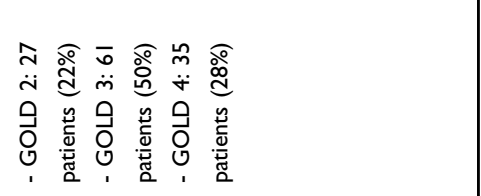 & 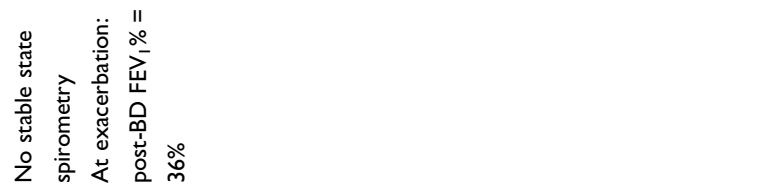 \\
\hline 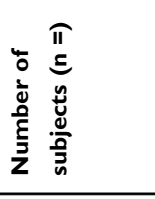 & 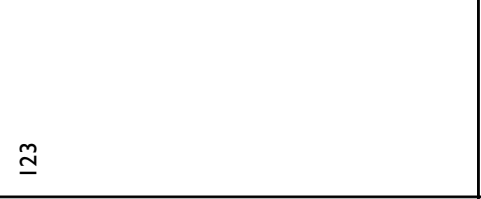 & $g$ \\
\hline 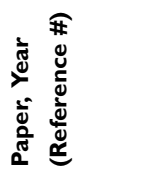 & 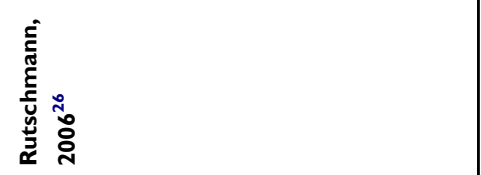 & 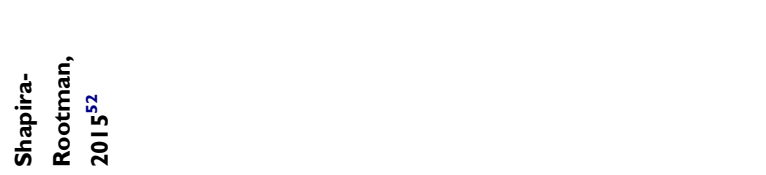 \\
\hline
\end{tabular}




\begin{tabular}{|c|c|c|}
\hline a & a & in \\
\hline 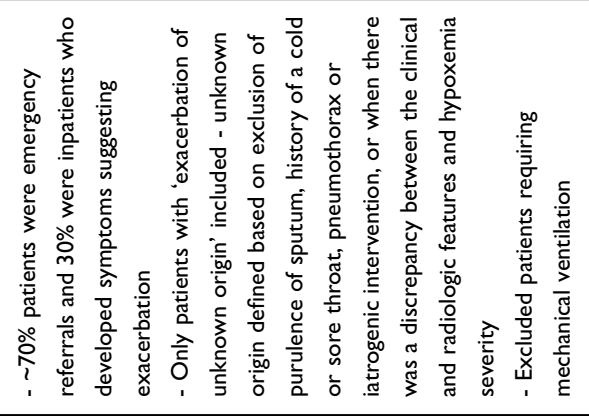 & 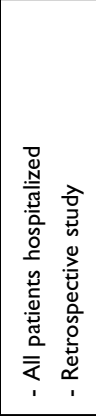 & 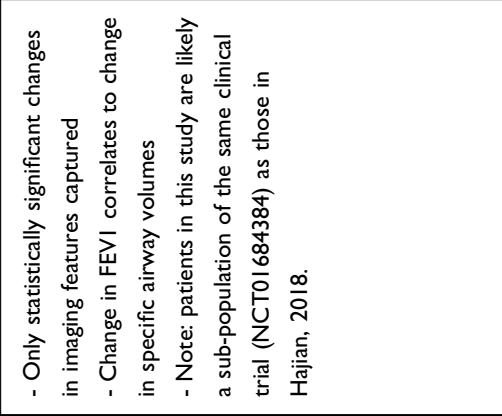 \\
\hline 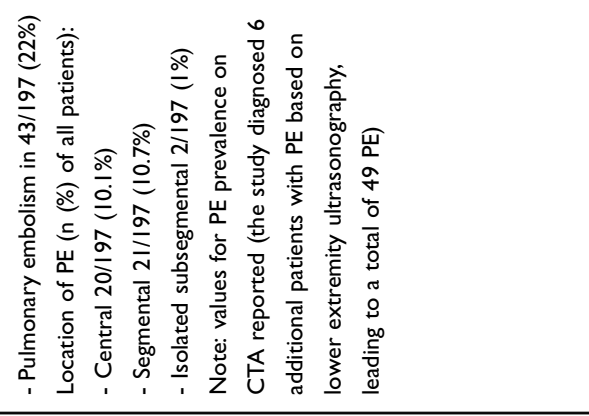 & 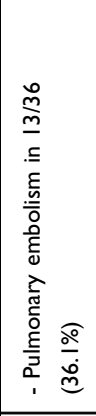 & 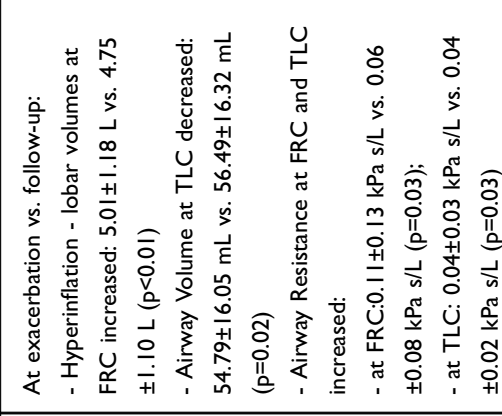 \\
\hline 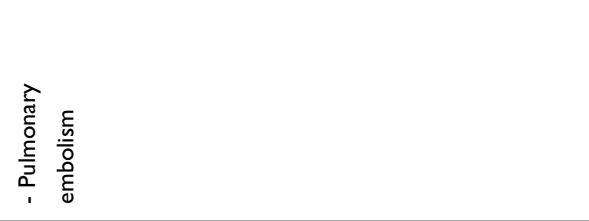 & 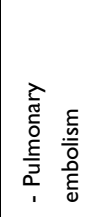 & 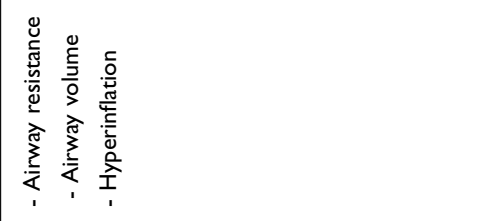 \\
\hline 吕 & \begin{tabular}{|l}
$\stackrel{\circ}{\circ}$ \\
$\stackrel{\sigma}{\sigma}$
\end{tabular} & 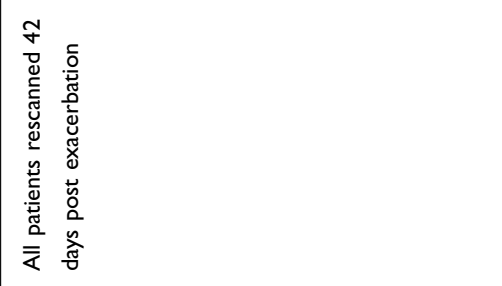 \\
\hline 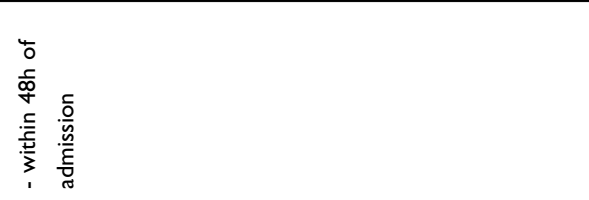 & & 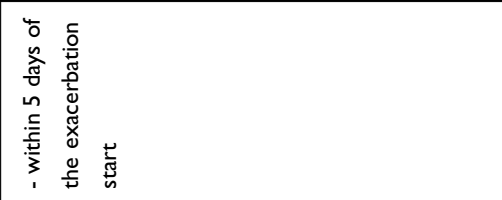 \\
\hline 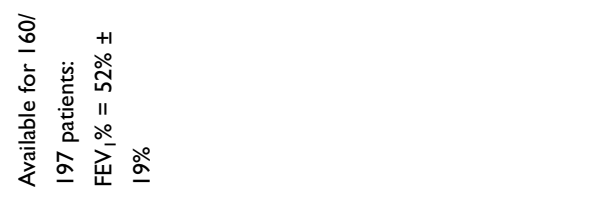 & 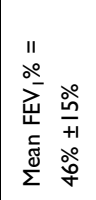 & 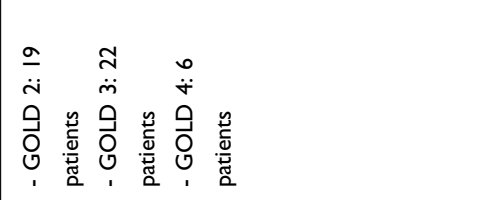 \\
\hline$\underline{\underline{a}}$ & $\stackrel{\circ}{\circ}$ & F \\
\hline 总 & 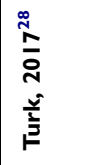 & 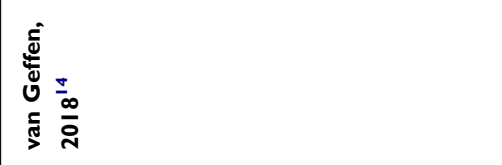 \\
\hline
\end{tabular}




\begin{tabular}{|c|c|c|}
\hline 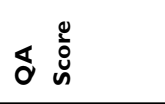 & $\mathrm{m}$ & n \\
\hline 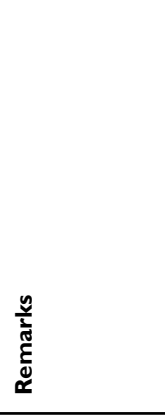 & 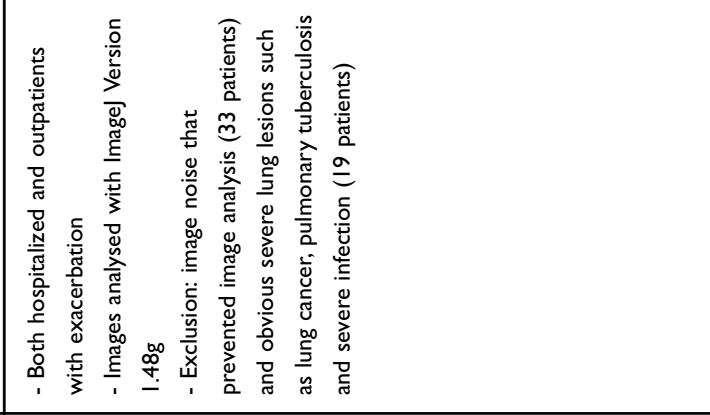 & 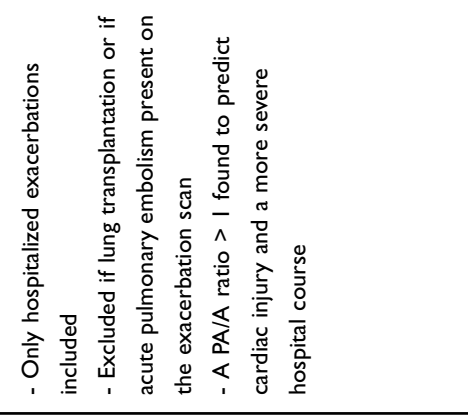 \\
\hline 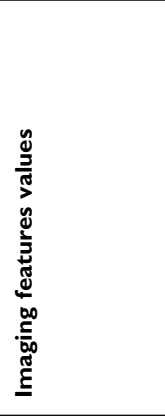 & 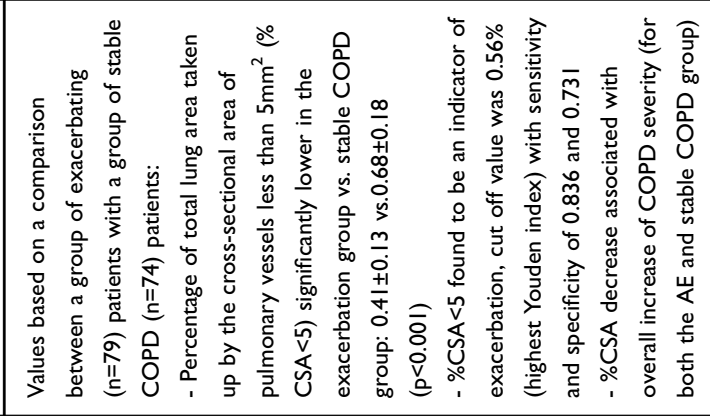 & 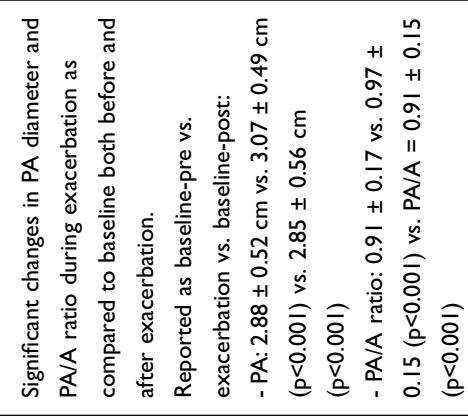 \\
\hline 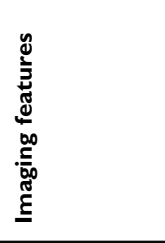 & 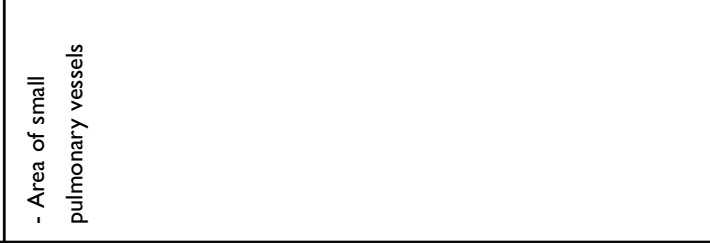 & 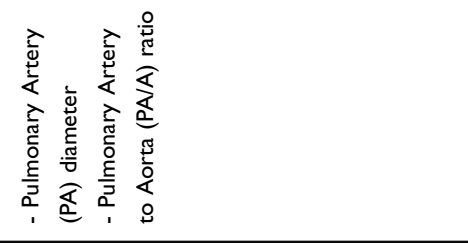 \\
\hline 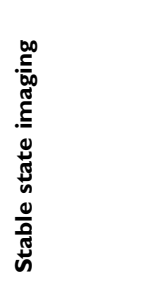 & 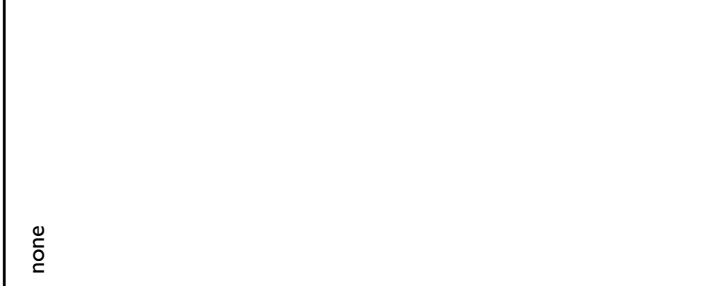 & 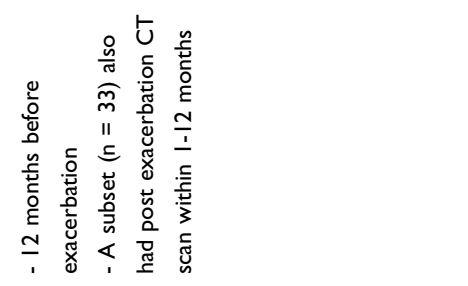 \\
\hline 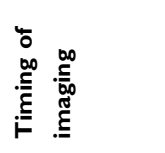 & 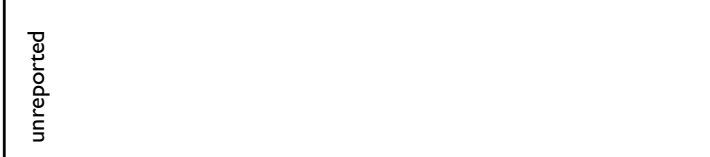 & 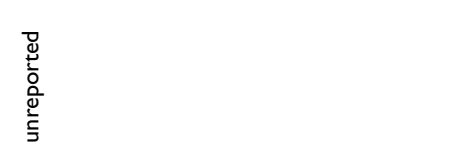 \\
\hline 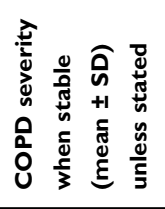 & 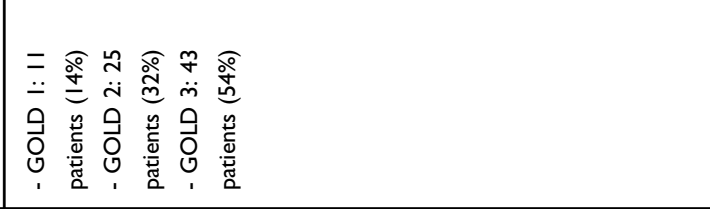 & 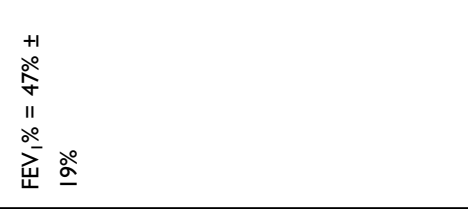 \\
\hline 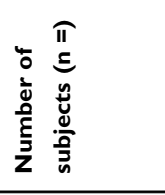 & 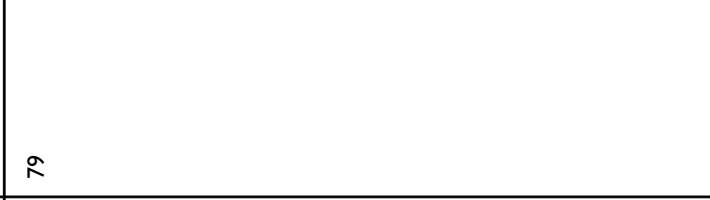 & $\stackrel{ \pm}{\underline{m}}$ \\
\hline 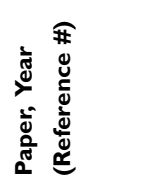 & 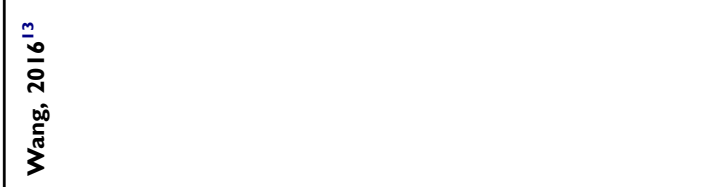 & 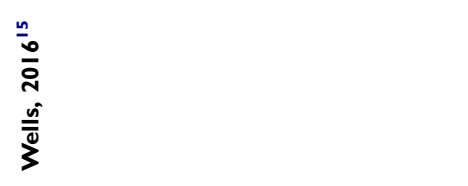 \\
\hline
\end{tabular}




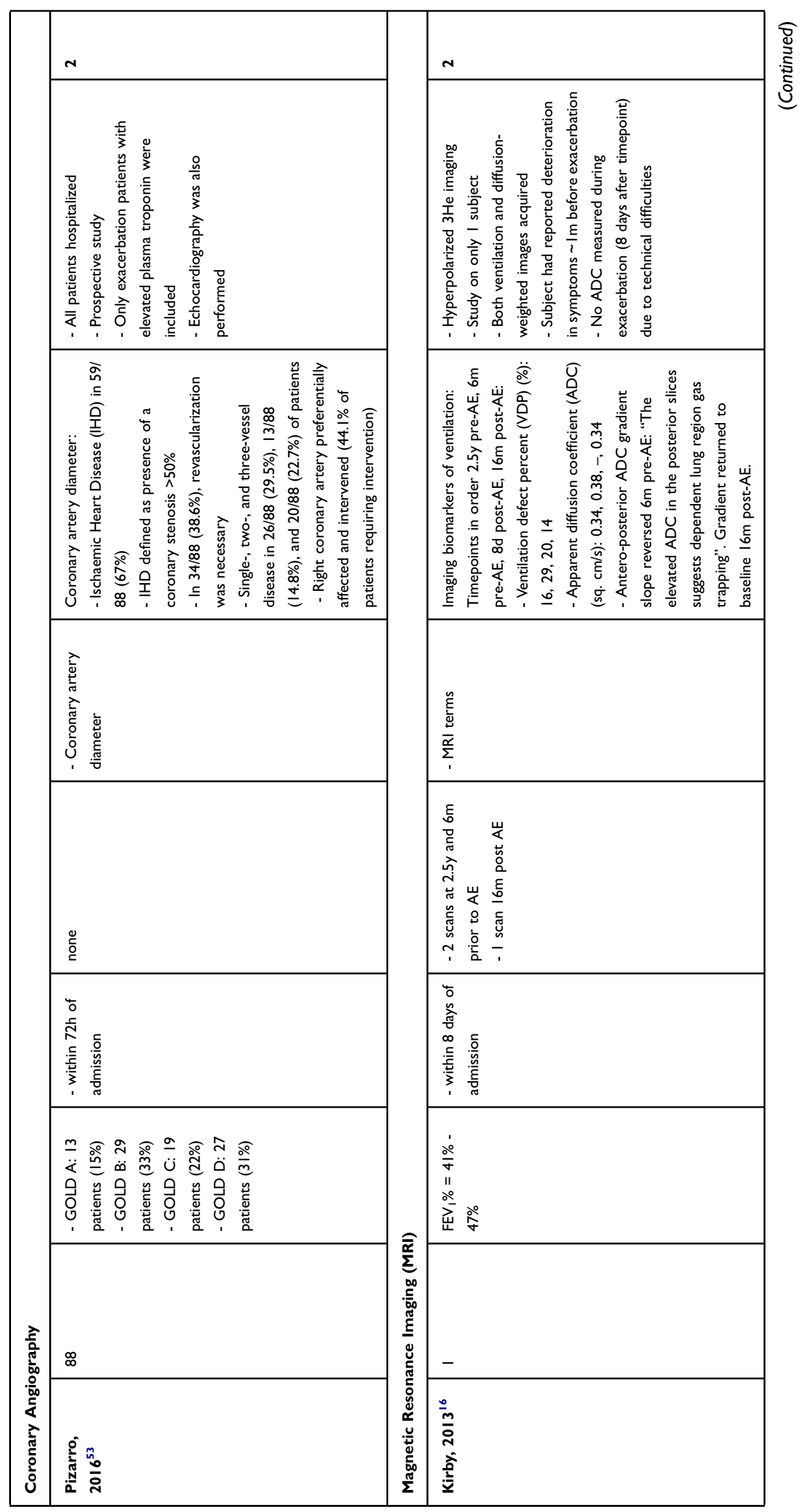




\begin{tabular}{|c|c|c|c|}
\hline ఠ & $m$ & & $\nabla$ \\
\hline 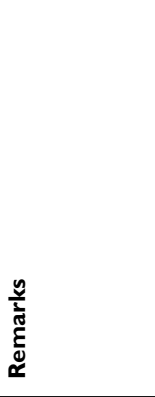 & 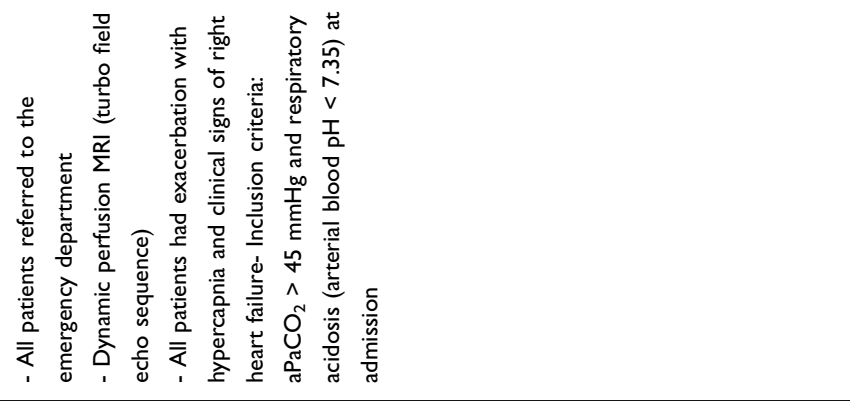 & & 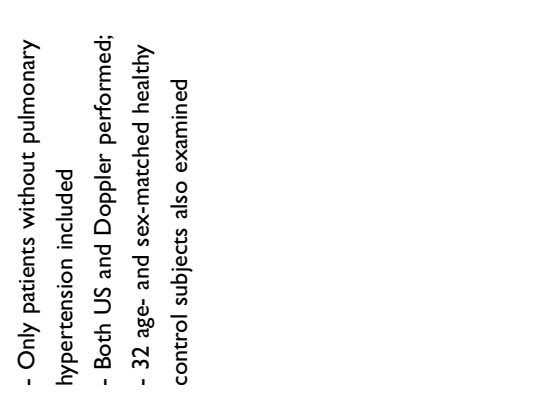 \\
\hline 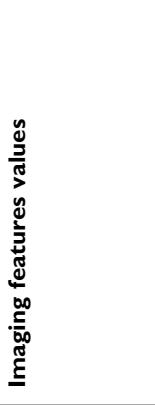 & 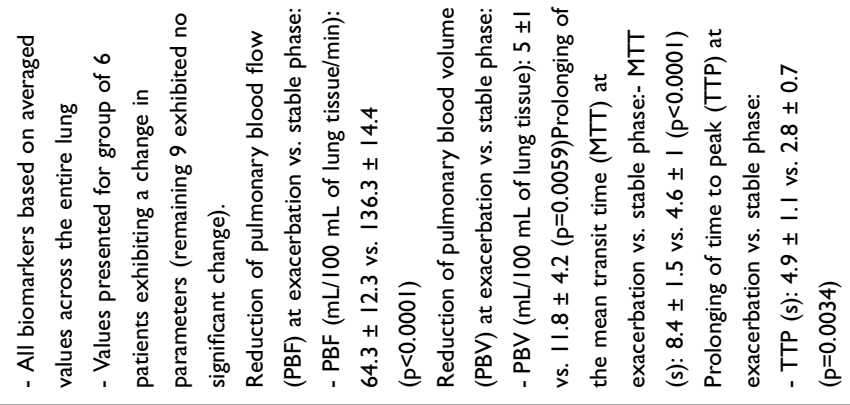 & & 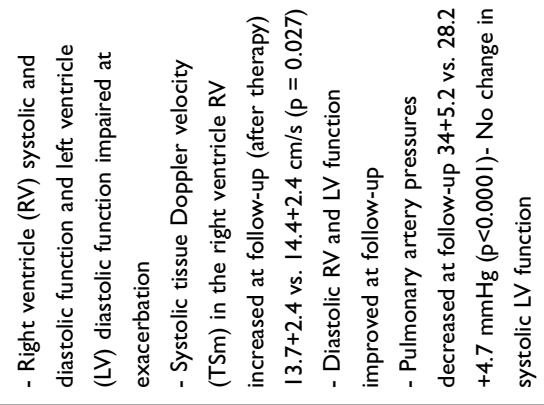 \\
\hline 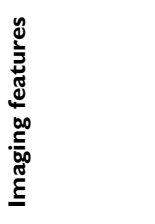 & $\begin{array}{l}\frac{\tilde{E}}{\tilde{E}} \\
\frac{\tilde{\Phi}}{\Sigma} \\
\frac{\tilde{\alpha}}{\Sigma}\end{array}$ & & 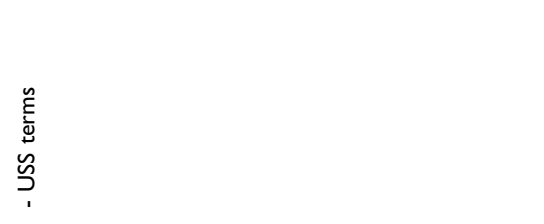 \\
\hline 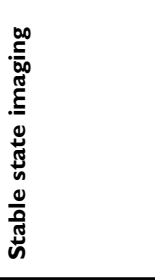 & 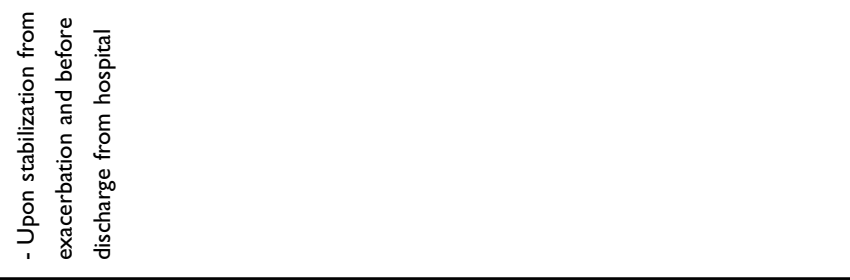 & & 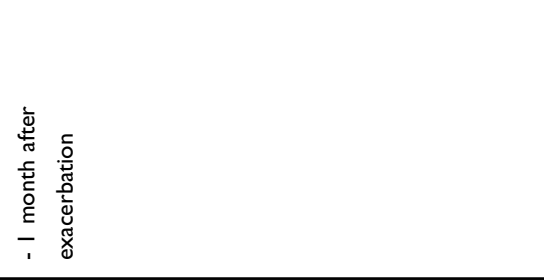 \\
\hline 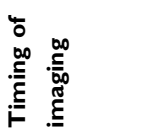 & 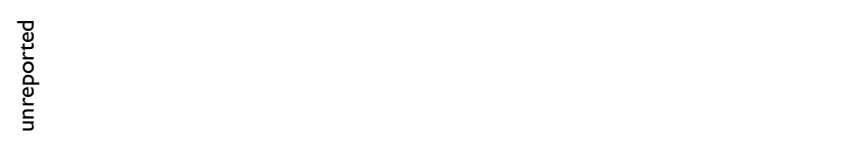 & & \\
\hline 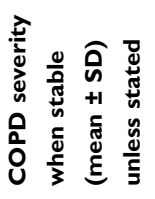 & $\begin{array}{l}\equiv \\
\stackrel{1}{\bar{\equiv}} \\
0 \\
0 \\
0 \\
\dot{z}\end{array}$ & & 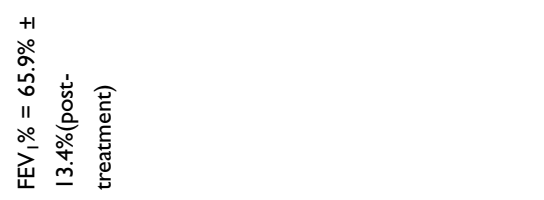 \\
\hline 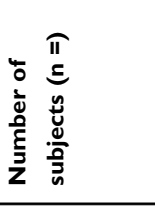 & $\underline{n}$ & 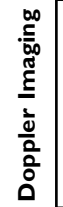 & $\widetilde{m}$ \\
\hline 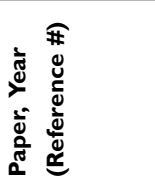 & 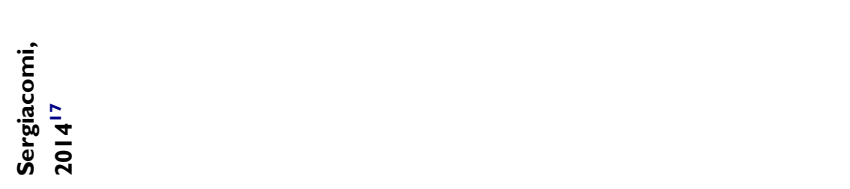 & 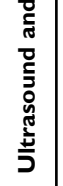 & 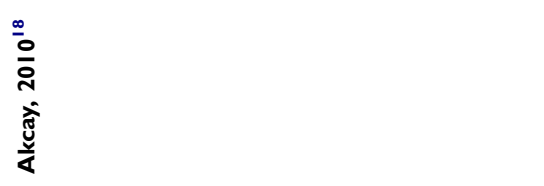 \\
\hline
\end{tabular}




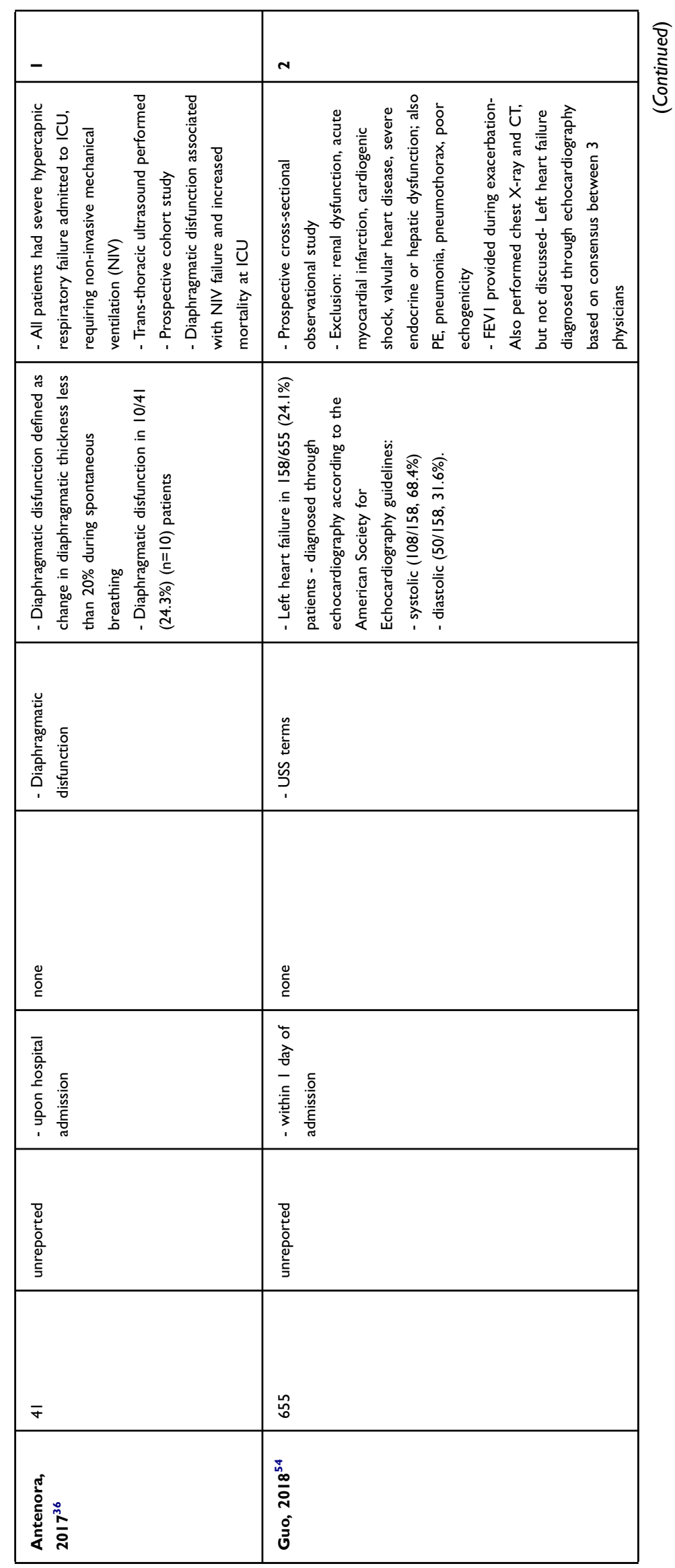




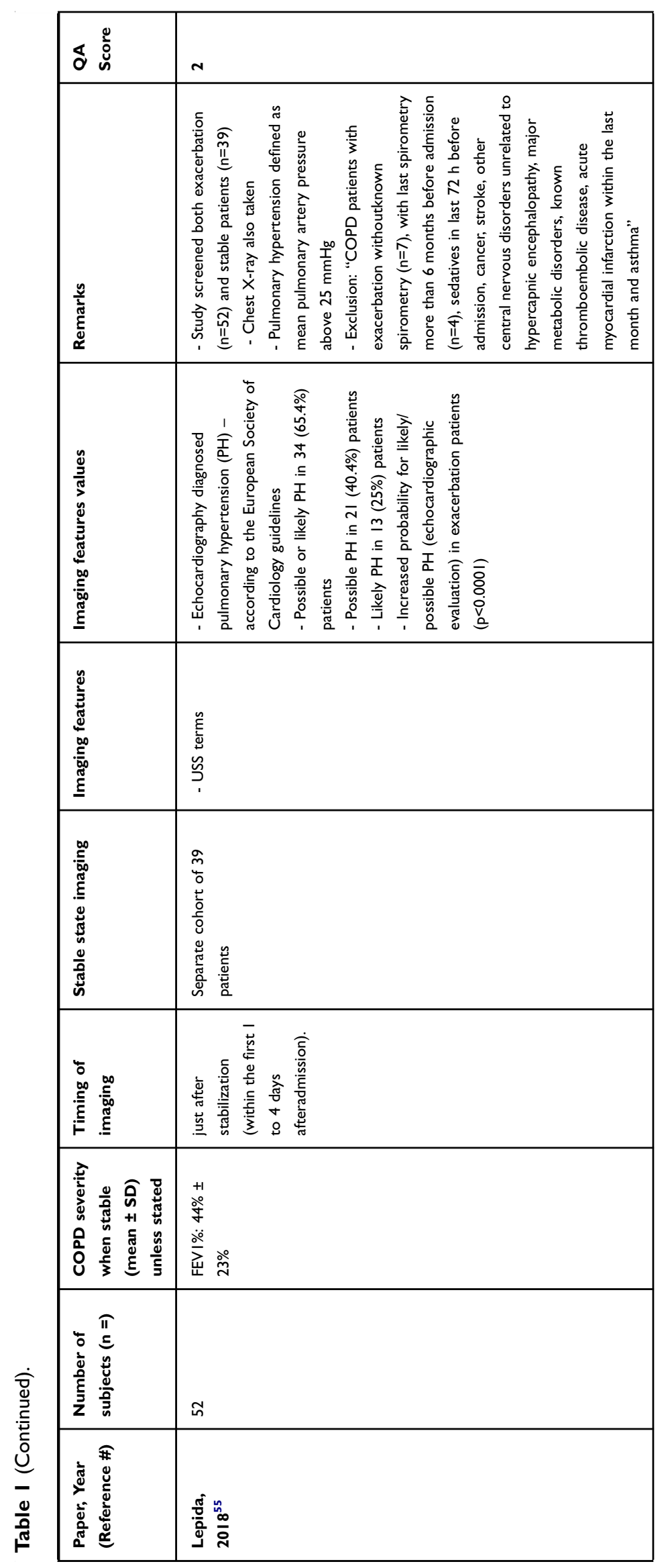




\begin{tabular}{|c|c|}
\hline$m$ & $m$ \\
\hline 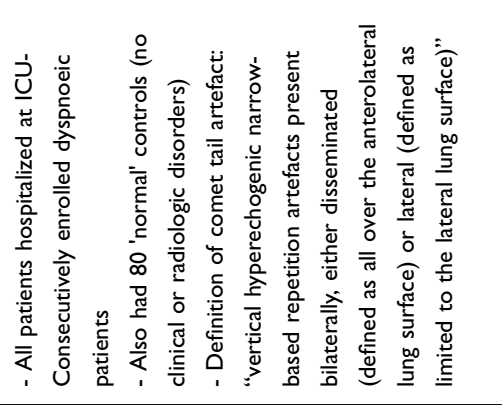 & 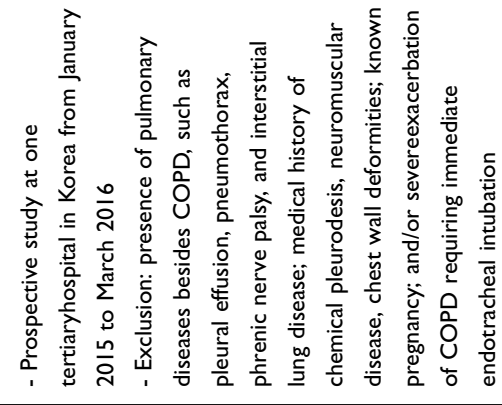 \\
\hline 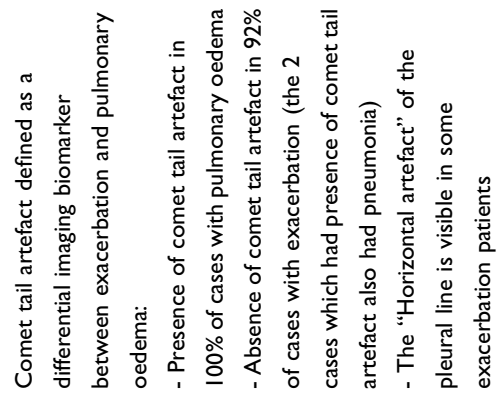 & 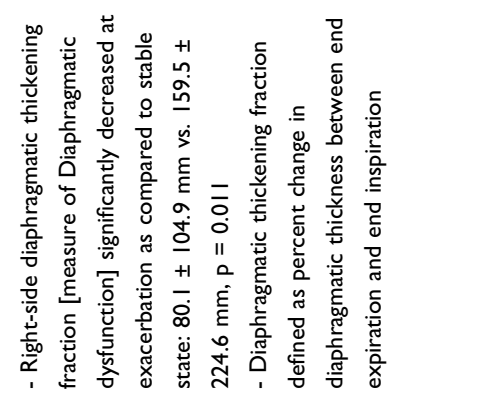 \\
\hline 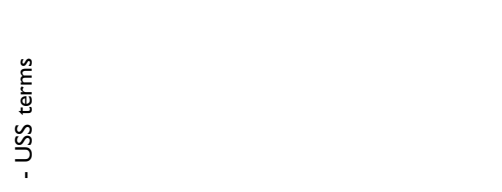 & 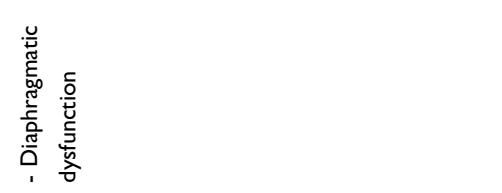 \\
\hline 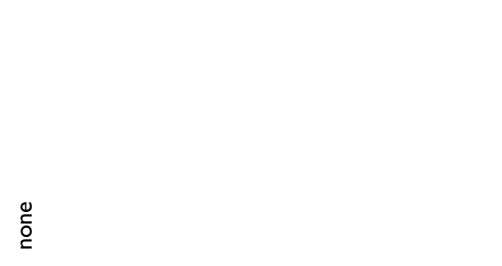 & 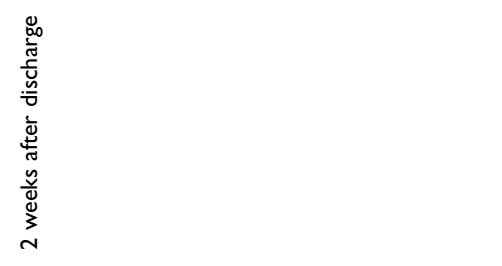 \\
\hline 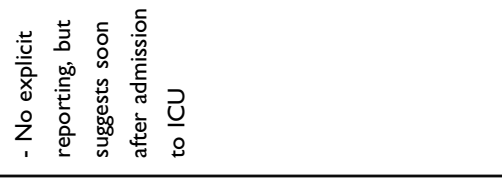 & 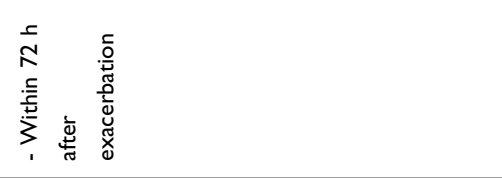 \\
\hline & 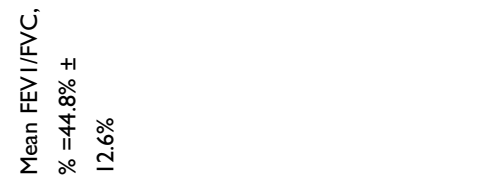 \\
\hline 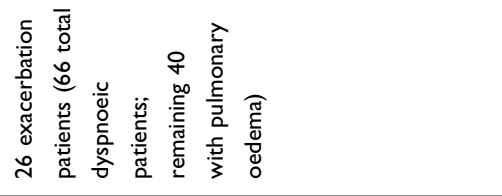 & 으 \\
\hline 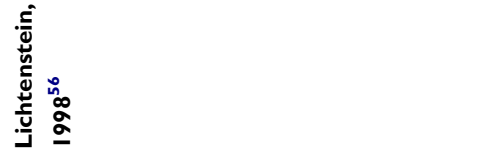 & 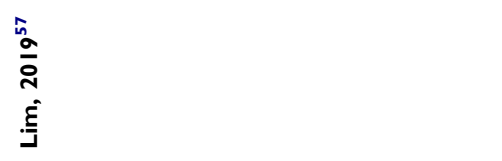 \\
\hline
\end{tabular}




\begin{tabular}{|c|c|}
\hline 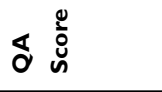 & - \\
\hline 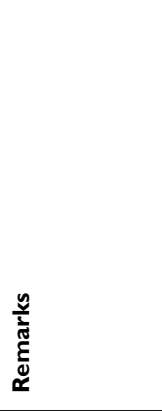 & 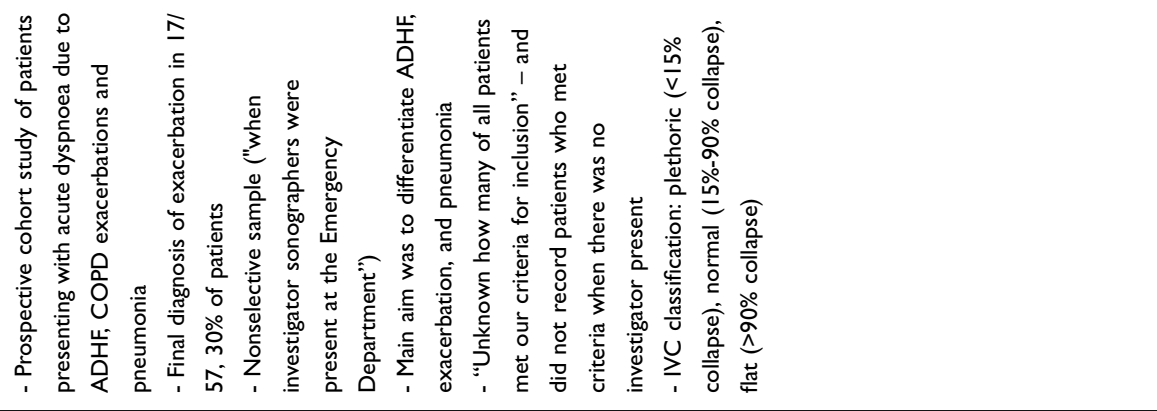 \\
\hline 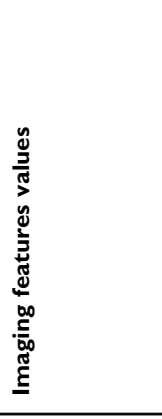 & 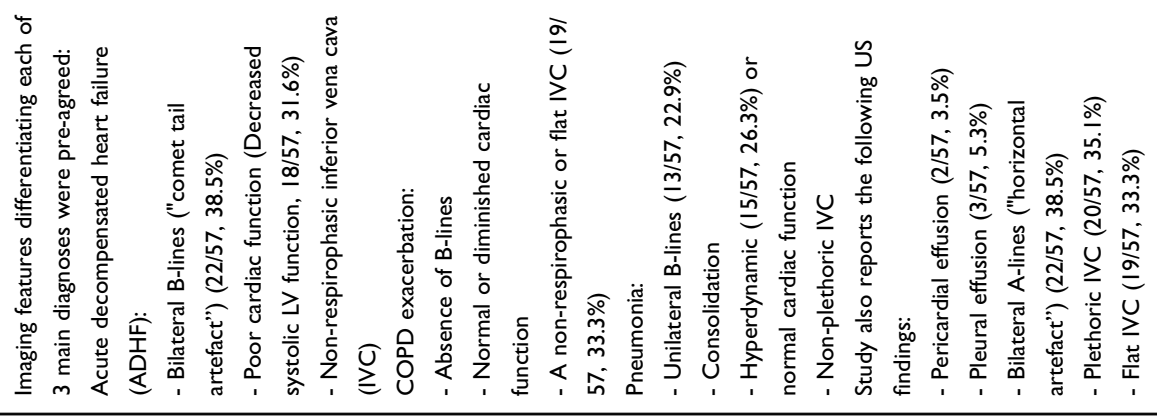 \\
\hline 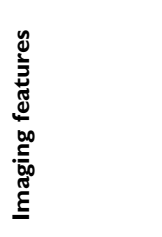 & 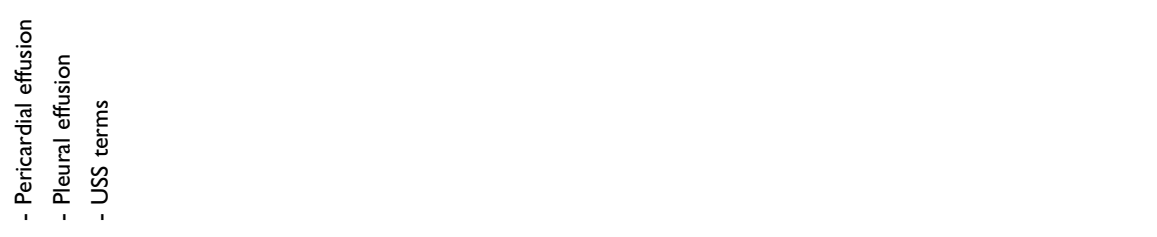 \\
\hline 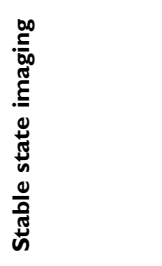 & $\begin{array}{l}\stackrel{0}{\circ} \\
\stackrel{0}{\circ}\end{array}$ \\
\hline 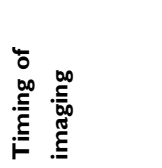 & 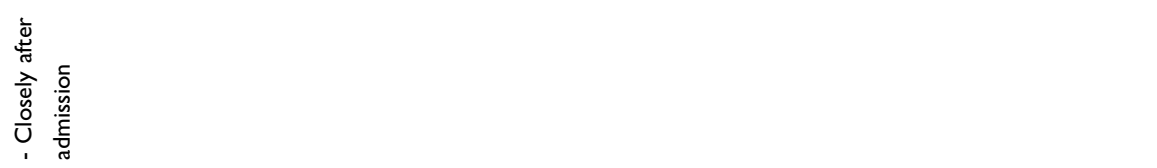 \\
\hline 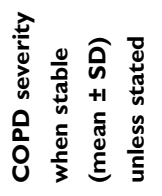 & \\
\hline 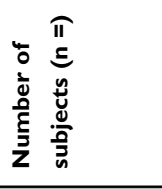 & 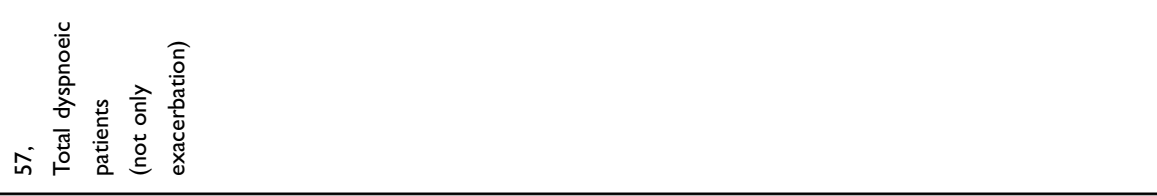 \\
\hline 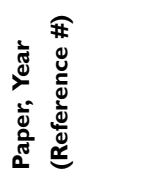 & 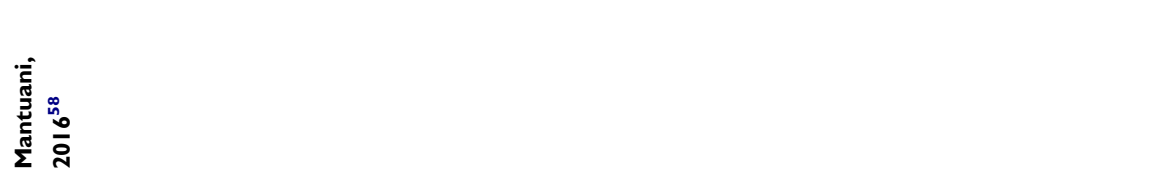 \\
\hline
\end{tabular}




\begin{tabular}{|c|c|}
\hline$N$ & N \\
\hline 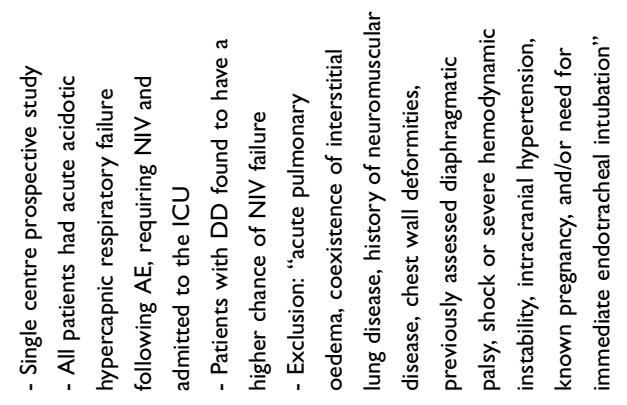 & 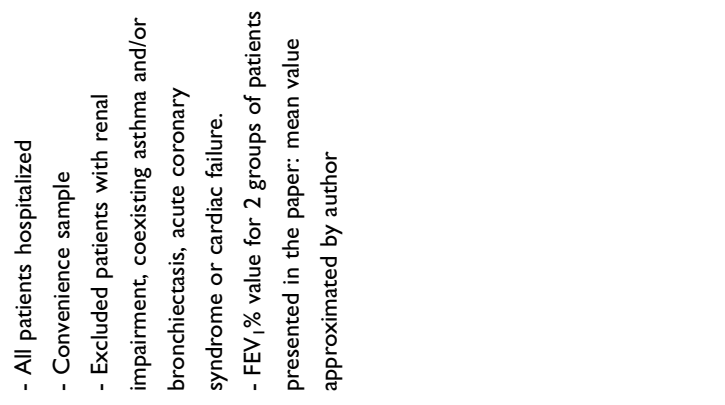 \\
\hline 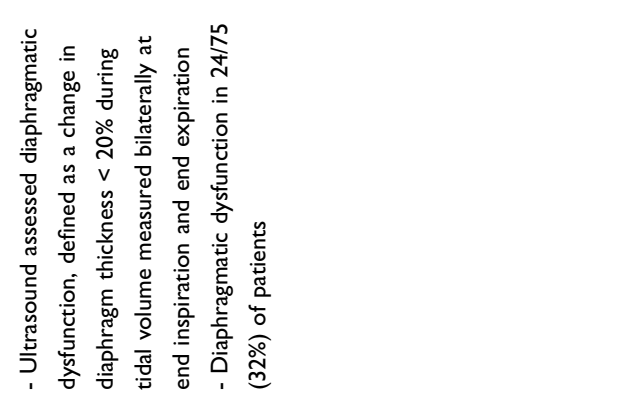 & 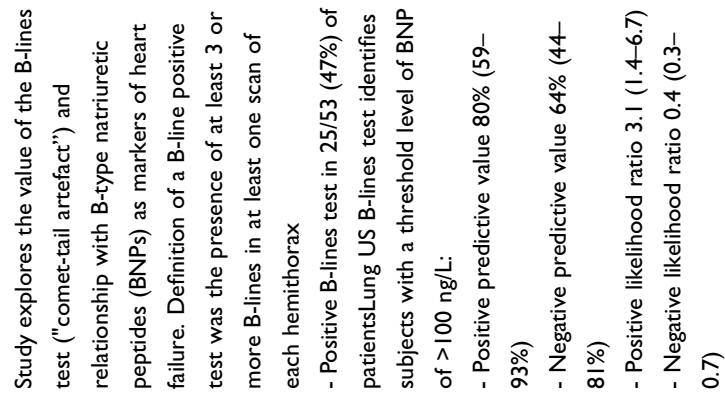 \\
\hline 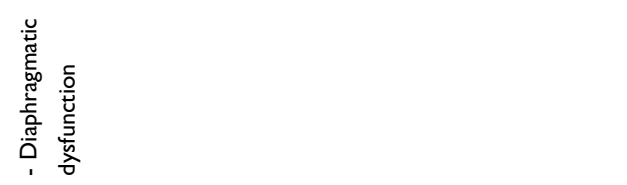 & 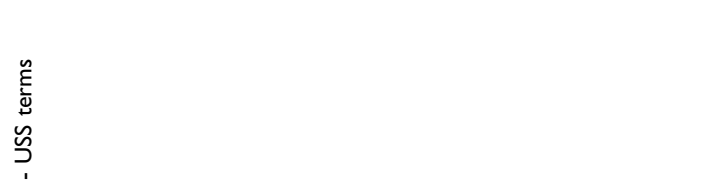 \\
\hline$\stackrel{0}{\stackrel{0}{0}}$ & $\begin{array}{l}\stackrel{0}{0} \\
\stackrel{0}{\check{\sigma}}\end{array}$ \\
\hline 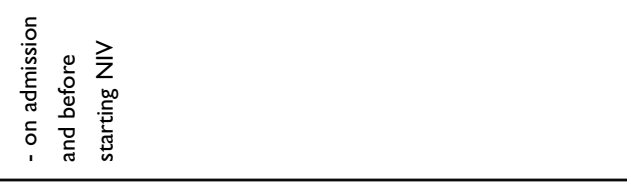 & 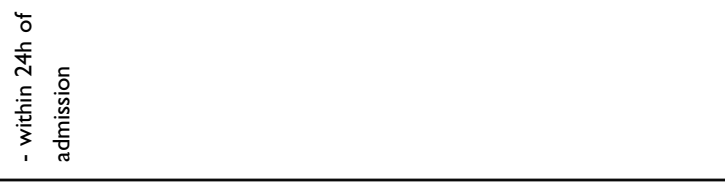 \\
\hline 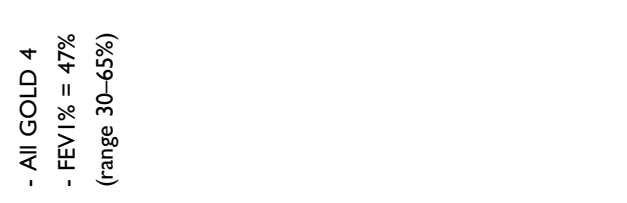 & 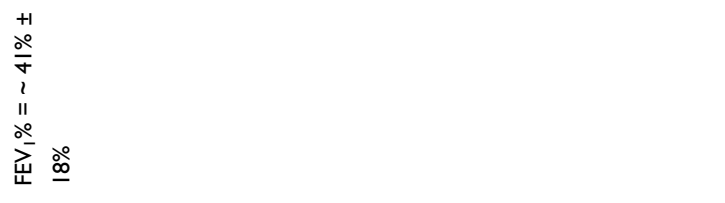 \\
\hline n & n \\
\hline 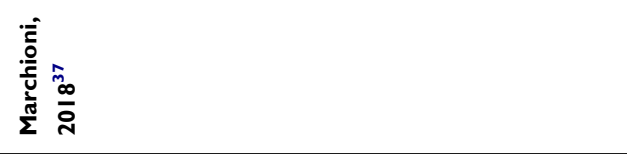 & 离 \\
\hline
\end{tabular}




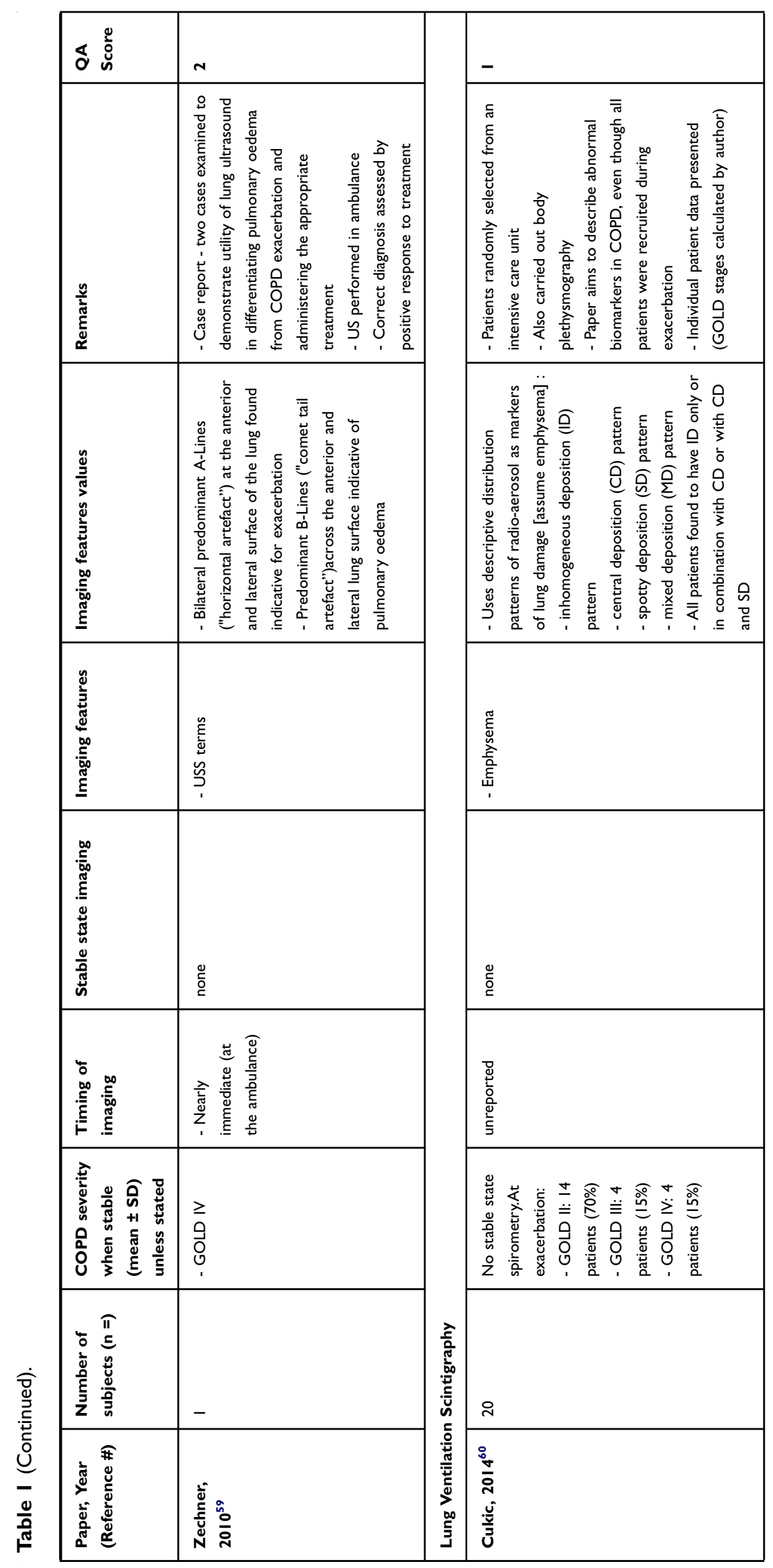




\begin{tabular}{|c|c|c|}
\hline$N$ & - & - \\
\hline 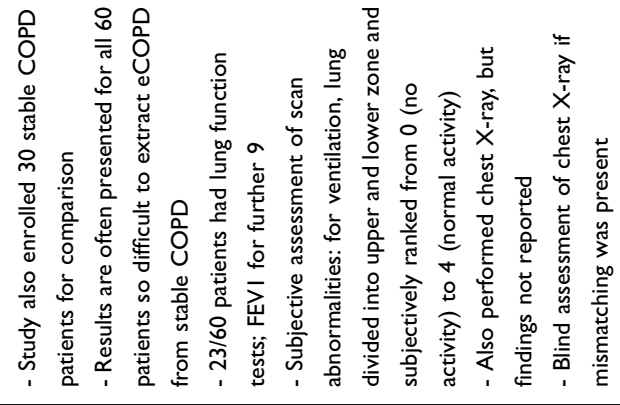 & 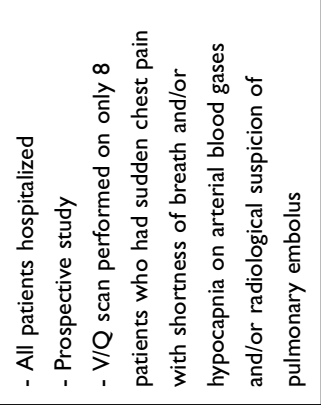 & 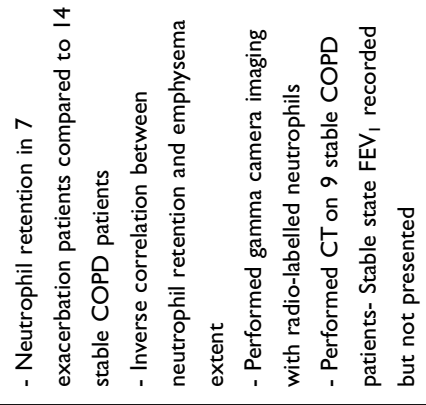 \\
\hline 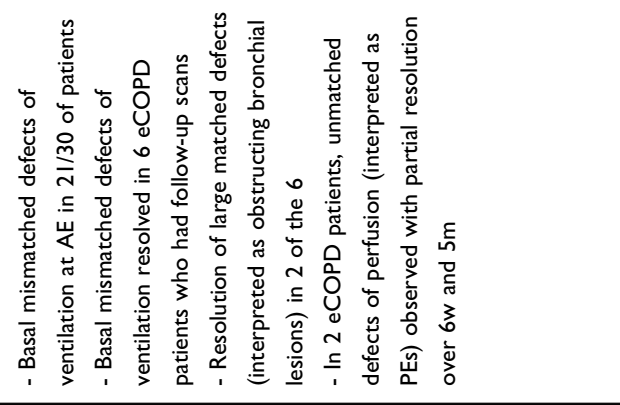 & 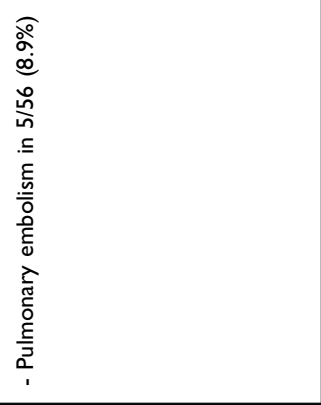 & 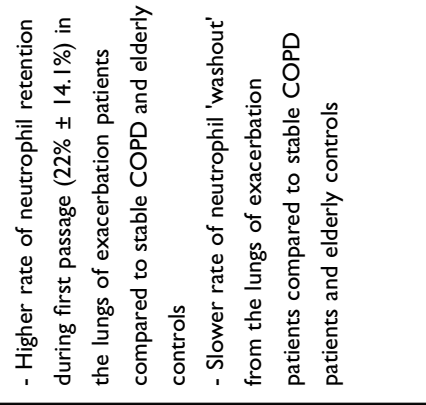 \\
\hline 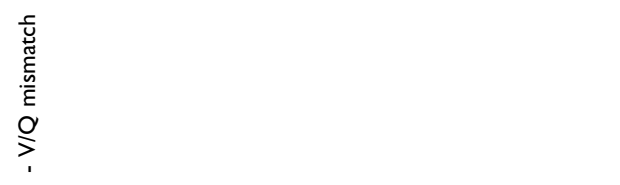 & 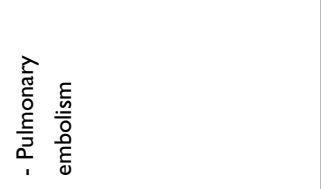 & 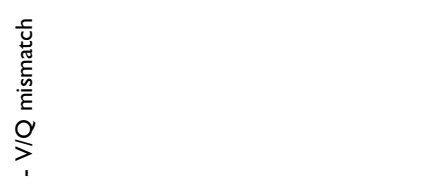 \\
\hline 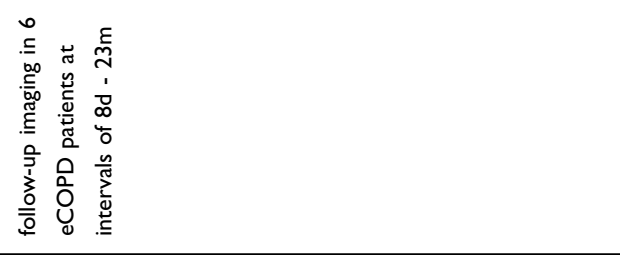 & $\begin{array}{l}\stackrel{0}{0} \\
\stackrel{0}{0}\end{array}$ & 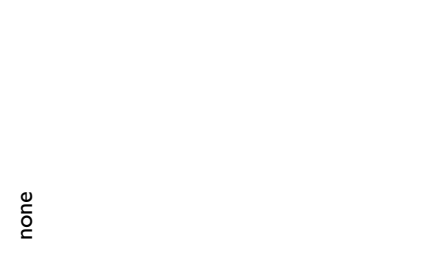 \\
\hline 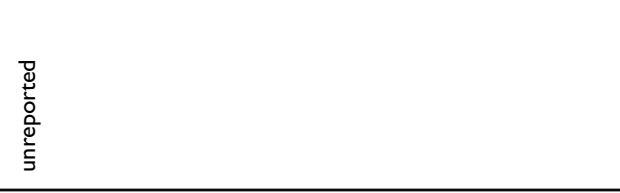 & 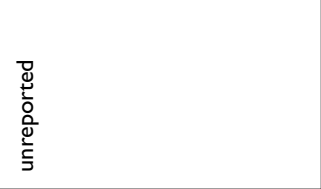 & 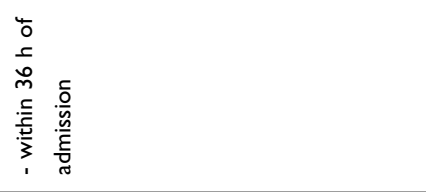 \\
\hline 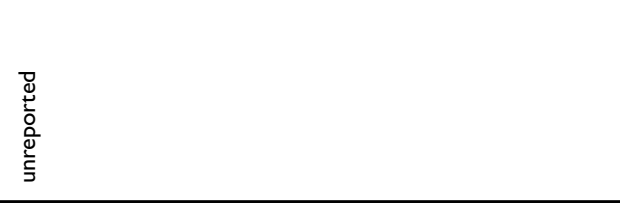 & 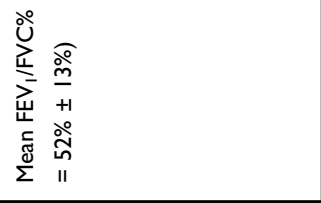 & 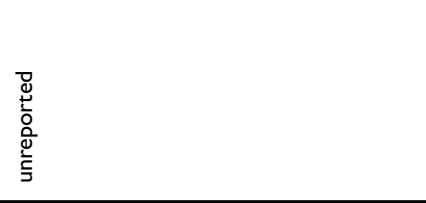 \\
\hline 요 & 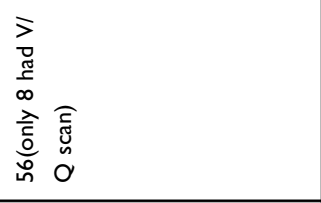 & $n$ \\
\hline 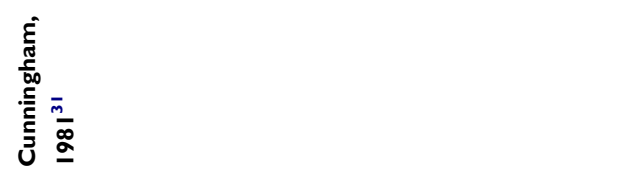 & 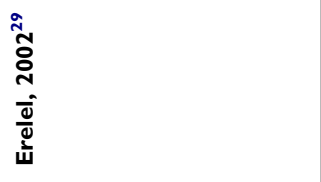 & $\begin{array}{l}\frac{\pi}{\sigma} \\
\frac{\sigma}{\alpha} \\
\frac{\hat{\alpha}}{\bar{\omega}} \\
\omega\end{array}$ \\
\hline
\end{tabular}




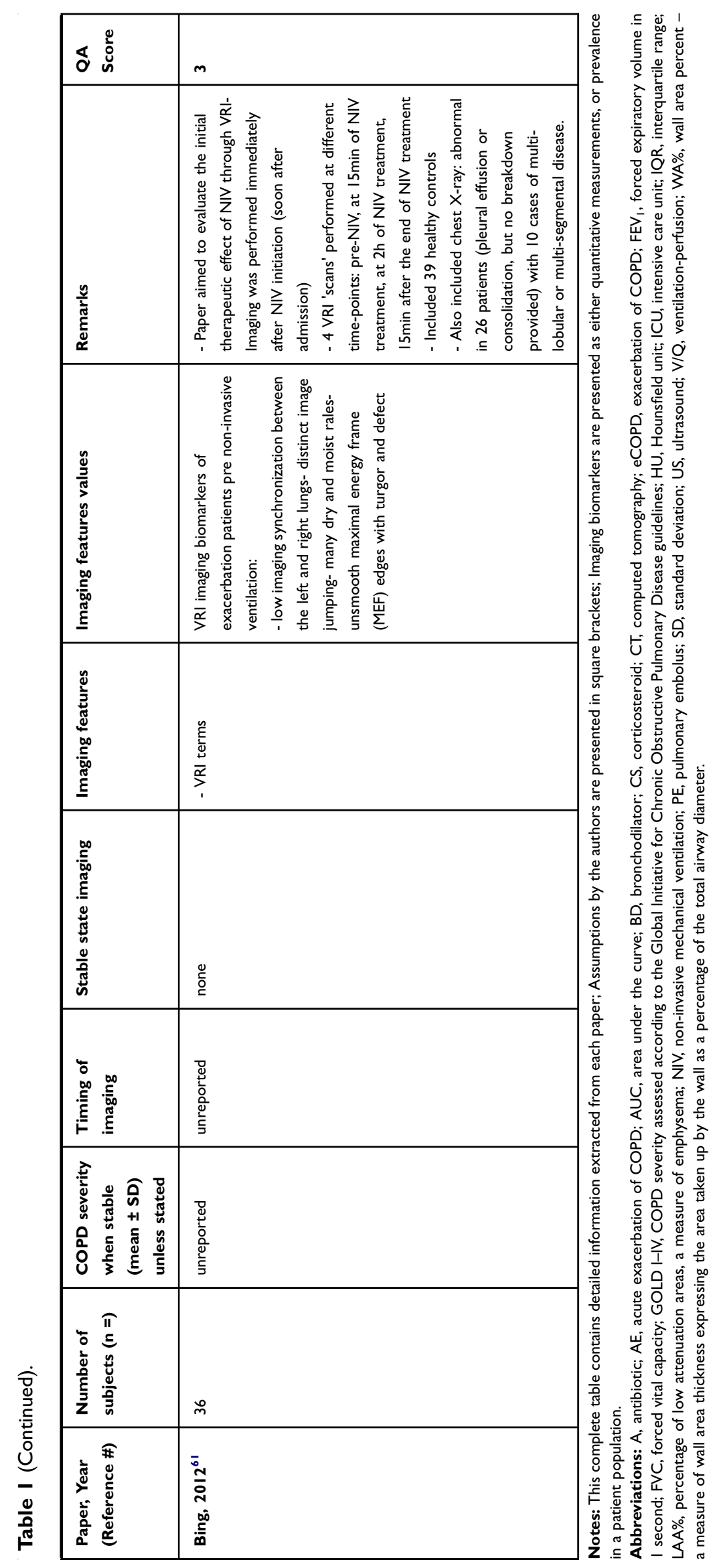




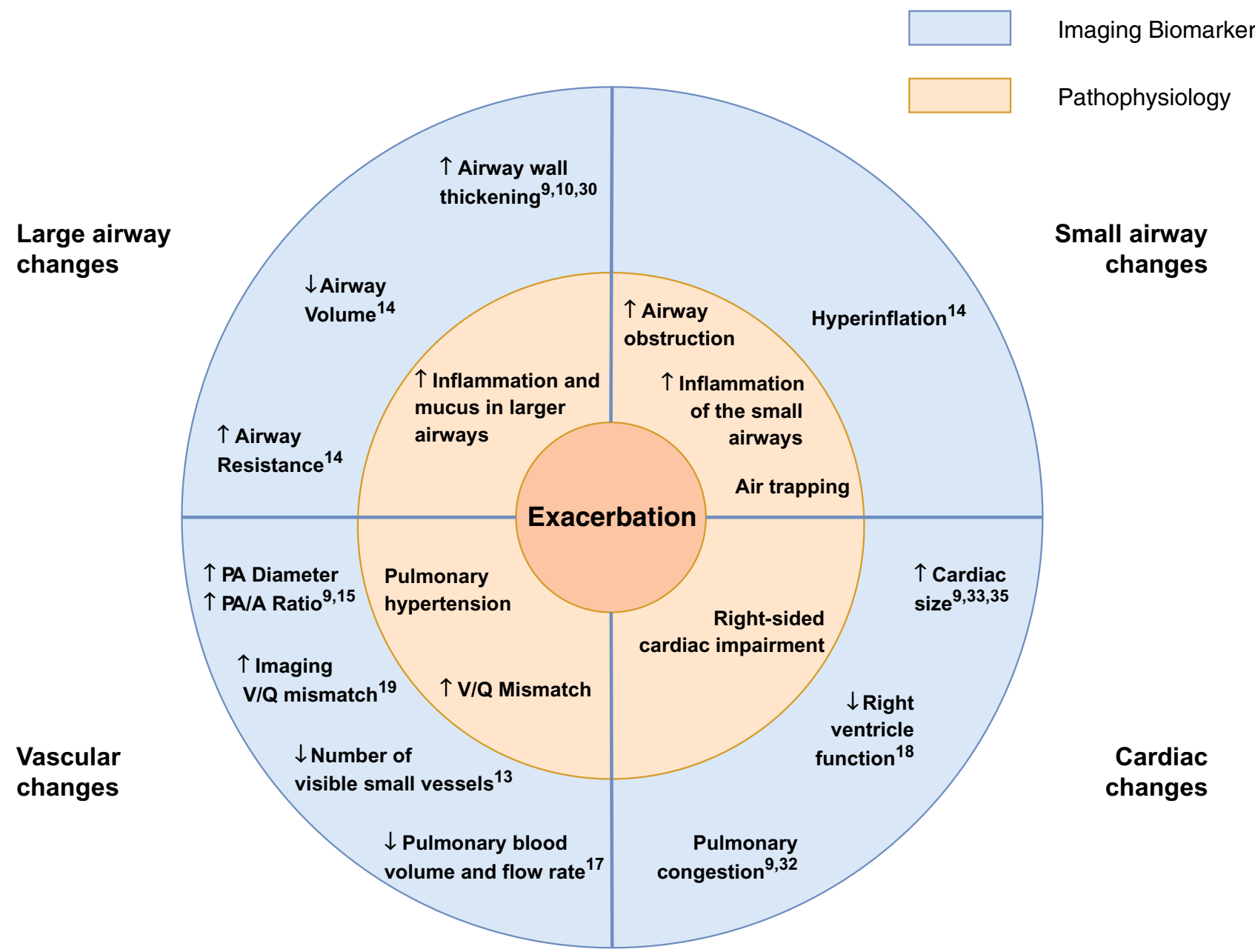

Figure 2 Summary: The Pathophysiology of a COPD Exacerbation in relation to Imaging Biomarkers. An exacerbation of COPD is associated with an increase in airway wall area and airway resistance, a decrease in airway calibre, hyperinflation, an increase in the diameter of the main pulmonary artery, a decrease in the number of visible small pulmonary vessels and cardiac enlargement. These features are suggestive of additional inflammation and mucus in the lumen of the airways, increased obstruction in the small airways and air trapping, pulmonary hypertension and right-sided cardiac impairment.

Abbreviations: PA, pulmonary artery; PA/A ratio, pulmonary artery to aorta ratio; V/Q mismatch, ventilation perfusion mismatch; $\uparrow$, increased; $\downarrow$, decreased.

(Table 1). These studies provide the most detailed information on imaging biomarkers of exacerbations.

Prominent features of exacerbations include changes in bronchial wall geometry: Cheng et $\mathrm{al}^{10}$ found increased wall area percent (WA\%) for the third generation airways and increased mean wall attenuation for the 3rd, 4th and 5th generation airways at exacerbation compared to follow-up. The increase in wall area as a percentage of the whole airway area may be an indicator of mucus or inflammatory infiltration within the lumen, while the increase in mean wall attenuation may represent inflammatory infiltration within the airway wall. Care must be taken with the latter parameter, however, since partial volume effect artefacts may lead to inaccurate measurements of attenuation of an airway wall with changing thickness. Bronchial wall geometry changes were also observed by Hackx et $\mathrm{al},{ }^{30}$ who noted bronchial wall thickening during an exacerbation, which decreased on follow-up. Van Geffen et $\mathrm{al}^{14}$ reported an increased imaging "airway resistance" both at total lung capacity (TLC) and functional residual capacity (FRC), derived by segmenting the airways and performing fluid dynamic modelling. This increased resistance could be explained by bronchoconstriction, airway wall changes and/or airway mucus plugging. Van Geffen et al additionally reported a decrease in total airway volume. Alotaibi et $\mathrm{al}^{9}$ also reported a high $(67.5 \%)$ prevalence of airway thickening on CT, although this latter study only examined a single time-point scan. 
Imaging features of the pulmonary vascular system also change at exacerbation. The study by Wells et al, ${ }^{15}$ which performed CT scans both up to 12 months before, at, and after exacerbation (the post-exacerbation scan was performed on a subsample of 33/134 patients), found statistically significant changes in the diameter of the main pulmonary artery (PA) and in the pulmonary artery to aorta (PA/A) ratio. The PA was enlarged during an exacerbation, leading to an increased $\mathrm{PA} / \mathrm{A}$ ratio, whilst the PA diameter both before and after an exacerbation was reduced compared to at exacerbation. This distension of the pulmonary artery may suggest a degree of pulmonary arterial hypertension. Wang et $\mathrm{al}^{13}$ studied the number of small pulmonary blood vessels visible during exacerbation compared to stable COPD. The group segmented out blood vessels with a diameter $5-10 \mathrm{~mm}$, and those with a diameter $<5 \mathrm{~mm}$, and defined metrics for the percentage of the total cross-sectional area of the lung taken up by each of the two vessel sizes $-\% \mathrm{CSA}_{<5}$ and $\% \mathrm{CSA}_{5-10}$. Interestingly, their results showed that $\% \mathrm{CSA}_{<5}$ was significantly decreased at exacerbation, without a decrease in $\% \mathrm{CSA}_{5-10}$. It should be noted, however, that the study did not scan patients at different time-points, but rather used two patient groups - one experiencing exacerbation and the other being stable COPD. The finding of fewer visible small blood vessels may represent vasoconstriction - the decrease in $\% \mathrm{CSA}_{<5}$ may arise from smaller blood vessels in the lung narrowing during an exacerbation and falling below the resolution of CT. The authors also observed that reduction in \%CSA was associated with increased COPD severity.

A further feature which changed between exacerbation and baseline was an increase in hyperinflation at FRC during exacerbation, quantified by van Geffen et al. ${ }^{14} \mathrm{~A}$ further study by the same group (on a subset of the same cohort), performed by Hajian et $\mathrm{al}^{19}$ also reported a significant improvement in the ventilationperfusion ratio (V/Q) from exacerbation to baseline. The study quantified "imaging $\mathrm{V} / \mathrm{Q}$ " by using lobar volume changes at TLC and FRC as a surrogate for ventilation and blood vessel density as a surrogate for perfusion. The V/Q improvement on recovery was mainly governed by improvement in the ventilation component, suggesting decreasing hyperinflation. There was no evidence of changes in emphysema extent (measured as LAA\%), measured in two studies by Cheng et al. ${ }^{10,11}$ Lung hyperinflation is an imaging biomarker directly related to increased air trapping, which can be a consequence of increased dysfunction of the small airways or emphysema. The absence of significant changes in the extent of emphysema, though, suggests that an exacerbation does not directly lead to alveolar destruction.

Taken together, the CT studies indicate that exacerbations of COPD are events which likely manifest primarily in the airways and vasculature, with secondary changes such as hyperinflation in the parenchyma. Both smaller and larger vessels and airways are likely to be involved.

\section{Chest X-Ray}

Fourteen articles employed chest X-ray (Table 1). The imaging features visible on a chest radiograph during an exacerbation were all reported as prevalence values for radiographic features. Of the five highest-ranking studies: ${ }^{9,32-35}$ Alotaibi et al reported an increased cardiac size in $16.2 \%$, Niksarlioglu et al in $19 \%$ and Shafuddin et al - in $25 \%$ of their populations respectively (however it is unlikely that this was an acute finding). Pleural effusion was reported in $11.6 \%$ of the population of Alotaibi et al and in $27 \%$ by Niksarlioglu et al Further prevalence findings in these two studies included pulmonary oedema in $15.5 \%,{ }^{9}$ bronchiectasis in $31.7 \%$ and emphysema in $60.3 \%{ }^{33}$ of patients - the latter two again are not likely to be acute. Johnson et al reported consolidation in $15 \%$ of patients and radiological features of congestive heart failure in $8 \%$. Williams et al found new pneumonic infiltrates in $42.6 \%$ of their population and Niksarlioglu et al in $54 \%$.

\section{MRI}

We found two studies of chest MRI at exacerbation of COPD. Kirby et $\mathrm{al}^{16}$ performed hyperpolarized ${ }^{3}$ Helium $^{16}$ imaging at four time-points to quantify ventilation defects at exacerbation, but their work can best be considered a pilot study since it only involved one subject. Sergiacomi et $\mathrm{al}^{17}$ used MRI to evaluate pulmonary perfusion and used an additional time-point early during recovery from exacerbation (before discharge from hospital) to compare imaging features. They found a reduction in pulmonary blood volume and pulmonary blood flow during an exacerbation, which is consistent with the CT studies described above. However, it should be noted that only six of 15 patients in the study exhibited changes.

\section{Ultrasound}

Ultrasound and Doppler imaging of the chest were performed in several studies which enrolled exacerbating 


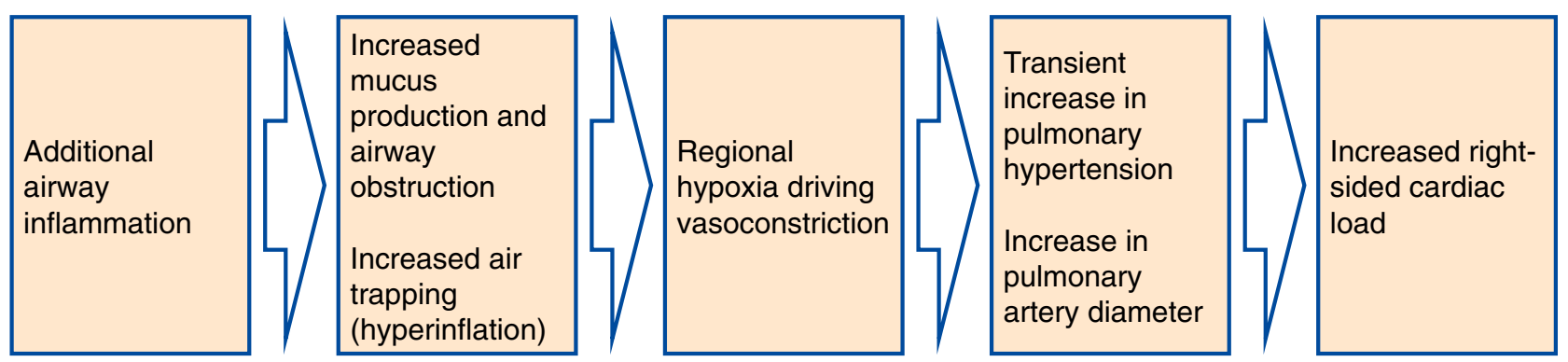

Figure 3 Hypothesis of the Pathophysiological Cascade Characteristic to an Exacerbation of COPD. Imaging features present during an exacerbation of COPD and their underlying pathophysiological processes motivate a hypothesis about the sequence of events in the lung which characterize an exacerbation. An exacerbation of COPD may be a cascade of events triggered by the onset of additional inflammation in the lumen of the airways, which leads to airway wall inflammation, bronchoconstriction and the production of excess mucus. This airway obstruction results in hyperinflation and the characteristic symptom of increased dyspnoea, and reactive vasoconstriction in small pulmonary vessels. In turn, this vasoconstriction leads to a transient increase in pulmonary arterial pressure, causing an enlargement of the pulmonary artery and right-sided cardiac dysfunction.

patients. Most of these, however, scored low on our quality score and did not perform follow-up imaging, focusing on using imaging features such as the "comet-tail artefact" to differentiate between pulmonary oedema and exacerbation of COPD in patients presenting to the emergency room with deterioration in symptoms. The highest-rated ultrasound research was the work by Akcay et $\mathrm{al}^{18}$ which followed patients one month after the exacerbation and found that during an exacerbation patients experienced impaired right ventricular systolic and diastolic function, as well as impaired left ventricular diastolic function. The study specifically excluded patients known to have pulmonary hypertension. Cardiac impairment improved at follow-up, which suggests that an exacerbation might indeed be associated with a transient increase in pulmonary hypertension. Antenora and Marchioni reported on the prevalence of diaphragm dysfunction at exacerbation, and associations with hyperinflation. ${ }^{36,37}$

\section{Other Imaging}

Several papers featuring nuclear medicine techniques such as Lung Ventilation Scintigraphy, and more exotic techniques such as Vibration Response Imaging (VRI) were included in the final synthesis. In general, they scored low on our quality score, did not perform longitudinal imaging and did not provide useful imaging biomarkers of exacerbation.

\section{Discussion}

We performed the first systematic review of imaging biomarkers at exacerbation of COPD. Such biomarkers might be useful in the objective diagnosis of exacerbation in the clinic and clinical trials, provide a mechanism for monitoring exacerbation treatment and recovery, and inform on the pathophysiological mechanisms of exacerbation.

Our results were strikingly consistent across studies, even though studies were heterogeneous in design. Most information was provided by $\mathrm{CT}$, which consistently describes increases in wall area measures at the time of exacerbation, air trapping, changes in the pulmonary vasculature in keeping with vasoconstriction and suggestive of pulmonary hypertension, and the absence of changes in measures of emphysema. These changes are supported by an MR study demonstrating reduced pulmonary blood volume and flow, ${ }^{17}$ and echocardiography studies suggesting right heart dysfunction. ${ }^{18}$

Other CT studies, and those using chest X-ray, reported the prevalence of specific radiological features in patients with suspected exacerbations. These often demonstrated additional or alternative co-morbidities responsible for the deterioration in symptoms, which would thus meet the definition of exacerbation, but in which imaging changed the primary diagnosis away from COPD exacerbation. Some of these studies described multiple imaging features $^{9,30}$ while others focused on identifying patients with a single possible alternative diagnosis such as PE. In both cases, the studies met our inclusion criteria for imaging during an exacerbation and consecutive enrolment of patients. Two large audits ${ }^{38,39}$ have demonstrated that the prevalence of consolidation on chest X-ray in COPD patients presenting with symptoms suggestive of exacerbation is $15-20 \%$ (and that this is associated with poorer outcomes). However, these scored low on our quality assessment as there was little available detail on patients or exacerbations. This emphasises the importance of 
developing imaging biomarkers of exacerbation: an imaging biomarker would enable a positive diagnosis of an exacerbation in clinical practice and research, rather than relying on the "clinical diagnosis of exclusion" model that is currently employed. CT would seem the best tool to achieve this. There were no positive diagnostic features on chest X-ray, whilst other bedside tests such as ultrasound have utility in the rapid exclusion of pulmonary oedema ("comet tail" artefacts), pneumothorax, pleural effusion and consolidation.

There are strengths to our review. Our comprehensive search strategy found 5,047 articles, of which 51 satisfied our inclusion criteria. While there is a significant body of literature on imaging features which correlate with, or sometimes predict exacerbations (for example the extent of emphysema $^{40}$ and PA/A ratio ${ }^{41}$ ), few studies have scanned patients during an exacerbation and compared this with the baseline state in the same patients. A potential limitation of the literature is that most (but not all) studies were performed in hospitalised patients and therefore the events studied typically represent severe presentations (a combination of COPD severity and severity of the exacerbation insult). Patients with mild and moderate community-treated exacerbations are rarely imaged during an exacerbation and there is a real need for further research in this area. Another potential limitation is that patients at exacerbation may find it difficult to enforce the protocols needed for good scan quality (eg, breath-hold). However, none of the studies reported significant artefacts in imaging. Furthermore, modern CT scanners acquire images in seconds with impressive quality, such that imaging during an exacerbation becomes increasingly feasible.

The included papers reported changes in different anatomical structures within the thorax and considered a variety of imaging biomarkers. While each of the articles focused on a particular aspect of exacerbation, systematically synthesizing the information has enabled us to generate a picture of the main features visible on medical imaging which characterize an exacerbation of COPD. These, together with their underlying pathophysiological processes, are summarised in Figure 2. One interpretation of the observed imaging characteristics of exacerbation, presented in Figure 3 is that an exacerbation of COPD represents a cascade of events triggered by the onset of additional inflammation in the airways, which leads to airway wall inflammation, bronchoconstriction and the production of excess mucus. This airway obstruction results in hyperinflation, and reactive vasoconstriction in small pulmonary vessels. In turn, the vasoconstriction leads to a transient increase in pulmonary arterial pressure, causing enlargement of the pulmonary artery and rightsided cardiac effects. There may be alternative explanations of the interrelatedness of the pathophysiological processes we have described.

For development as a tool in clinical trials, quantitative imaging measures are preferable to qualitative features. We did find quantitative measures of imaging features during exacerbation. Quantitative assessment of emphysema is already used in clinical practice to guide volume reduction interventions (but did not change at exacerbation). There are no existing quantitative imaging biomarkers of exacerbation or exacerbation recovery, and based on our work, plausible candidates might include assessment of airway wall area and volume, blood vessel calibre and volume, and gas trapping.

For use in the routine diagnosis and management of exacerbations, imaging biomarkers present an opportunity to achieve a quantitative, positive diagnosis of exacerbation, which could lead to reduction in misdiagnosis and an improved understanding of the pathophysiology of exacerbations. Quantitative exacerbation imaging metrics may also provide a tool to study exacerbation phenotypes which could further motivate development of novel treatments and permit a personalised medicine approach to exacerbation management.

A surprising finding was the absence of studies examining functional imaging such as positron-emission tomography. Specific cell-labelling techniques have demonstrated increased neutrophil retention within the lung at exacerbation, ${ }^{42}$ and slower rates of neutrophil wash-out compared to stable COPD and control subjects without COPD.

In conclusion, we have performed the first systematic review of imaging biomarkers at exacerbation of COPD. We find that an exacerbation is characterised by airway calibre and wall changes, hyperinflation, pulmonary vasoconstriction and pulmonary arterial hypertension. Exacerbation imaging biomarkers lay an important foundation for the objective diagnosis of exacerbation in the clinic and clinical trials, as well as for monitoring of exacerbation treatment and recovery.

\section{Acknowledgments}

BAR would like to express his gratitude to Jennifer Ford, Evidence Services Librarian at the Royal Free Medical Library for the support and guidance in planning the systematic review and preparing the search strategies. 


\section{Funding}

BAR is supported by the EPSRC Centre For Doctoral Training in Medical Imaging with grant EP/L016478/1 and an industrial CASE studentship with funding from GlaxoSmithKline, with studentship agreement number BIDS3000032413. BAR is also holding an Industrial Fellowship from the Royal Commission for the Exhibition of 1851. ALY is supported by an MRC Skills Development Fellowship. JJ was supported by a Wellcome Trust Clinical Research Career Development Fellowship 209553/Z/17/Z. APC and FJW are employees of GlaxoSmithKline. SL is a consultant to GlaxoSmithKline.

\section{Disclosure}

BAR reports grants from GlaxoSmithKline. He has recently won an Industrial Fellowship from the Royal Commission for the Exhibition of 1851. ALY reports grants from the EPSRC and MRC during the conduct of this study. JJ reports personal fees from Boehringer Ingelheim and from Roche outside the submitted work. SL reports personal fees from GlaxoSmithKline during the conduct of the study and personal fees from GE Healthcare outside the submitted work. APC and FJW are employees and shareholders of GlaxoSmithKline. DJH reports grants from GlaxoSmithKline, grants from EPSRC during the conduct of the study; shareholder and founder in IXICO PLC and shareholder in Vision RT until May 2018, personal fees from HawkesMedTech Ltd and he is a founder of HawkesMedTech Ltd, outside the submitted work. JRH reports he supervised BAR's $\mathrm{PhD}$ which is funded by GlaxoSmithKline, and that during the conduct of the study received grants, personal fees and non-financial support from pharmaceutical companies which make medicines to treat COPD outside the submitted work.

\section{References}

1. Anzueto A. Impact of exacerbations on COPD. Eur Respir Rev. 2010;19(116):113-118. doi:10.1183/09059180.00002610

2. Vogelmeier CF, Criner GJ, Martinez FJ, et al. Global strategy for the diagnosis, management, and prevention of chronic obstructive lung disease 2017 report. GOLD executive summary. Am J Respir Crit Care Med. 2017;195(5):557-582. doi:10.1164/rccm.2017010218PP

3. Moher D, Liberati A, Tetzlaff J, Altman DG. Preferred reporting items for systematic reviews and meta-analyses: the PRISMA statement Ann Intern Med. 2009;151(4):264. doi:10.7326/0003-4819-151-4-20 0908180-00135

4. Huang X, Lin J, Demner-Fushman D Evaluation of PICO as a knowledge representation for clinical questions. In: AMIA Annual Symposium proceedings. 2006. AMIA; 2006:359-363.
5. Rangelov B, Hurst J, Medical imaging at exacerbation of COPD PROSPERO: international prospective register of systematic reviews. CRD42018095417.

6. EndNote X8| Clarivate Analytics. Available from: https://endnote. com/. Accessed December 18, 2018.

7. Ouzzani M, Hammady H, Fedorowicz Z, Elmagarmid A. Rayyan-a web and mobile app for systematic reviews. Syst Rev. 2016;5(1):210. doi:10.1186/s13643-016-0384-4

8. Luchini C, Stubbs B, Solmi M, Veronese N. Assessing the quality of studies in meta-analyses: advantages and limitations of the Newcastle Ottawa Scale. World J Meta-Analysis. 2017;5(4):80. doi:10.13105/wjma. v5.i4.80

9. Alotaibi NM, Chen V, Hollander Z, et al. Phenotyping COPD exacerbations using imaging and blood-based biomarkers. Int $J$ COPD. 2018;13:217-229. doi:10.2147/COPD.S152484

10. Cheng T, Wan HY, Cheng QJ, et al. Computed tomography manifestation of acute exacerbation of chronic obstructive pulmonary disease: A pilot study. Exp Ther Med. 2016;11(2):519-529.

11. Cheng T, Wan HY, Cheng QJ, et al. Obvious emphysema on computed tomography during an acute exacerbation of chronic obstructive pulmonary disease predicts a poor prognosis. Intern Med $\mathrm{J}$. 2015;45(5):517-526.

12. Leong P, Tran A, Rangaswamy J, et al. Expiratory central airway collapse in stable COPD and during exacerbations. Respir Res. 2017;18(1): 163 .

13. Wang Z, Chen X, Liu K, et al. Small pulmonary vascular alteration and acute exacerbations of COPD: quantitative computed tomography analysis. Int J Chron Obstruct Pulmon Dis. 2016;11:1965-1971. doi:10.2147/COPD.S112651

14. van Geffen WH, Hajian B, Vos W, et al. Functional respiratory imaging: heterogeneity of acute exacerbations of COPD. Int J Chron Obstruct Pulmon Dis. 2018;13:1783-1792. doi:10.2147/COPD.S152463

15. Michael Wells J, Morrison JB, Bhatt SP, Nath H, Dransfield MT, Pulmonary artery enlargement is associated with cardiac injury during severe exacerbations of COPD. Chest. 2016;149(5):1197-1204. doi:10.1378/chest.15-1504

16. Kirby M, Kanhere N, Etemad-Rezai R, McCormack DG, Parraga G, Hyperpolarized helium-3 magnetic resonance imaging of chronic obstructive pulmonary disease exacerbation. $J$ Magn Reson Imaging. 2013;37(5):1223-1227. doi:10.1002/jmri.23896

17. Sergiacomi G, Taglieri A, Chiaravalloti A, et al. Acute COPD exacerbation: 3 T MRI evaluation of pulmonary regional perfusion-preliminary experience. Respir Med. 2014;108(6):875-882.

18. Akcay M, Yeter E, Durmaz T, et al. Treatment of acute chronic obstructive pulmonary disease exacerbation improves right ventricle function. Eur J Echocardiogr. 2010;11(6):530-536.

19. Hajian B, De Backer J, Vos W, et al. Changes in ventilation-perfusion during and after an COPD exacerbation: an assessment using fluid dynamic modeling. Int $J$ Chron Obstruct Pulmon Dis. 2018;13:833-842. doi:10.2147/COPD.S153295

20. Akpinar EE, Hosgun D, Akpinar S, Atac GK, Doganay B, Gulhan M, Incidence of pulmonary embolism during COPD exacerbation. J Bras Pneumol. 2014;40(1):38-45. doi:10.1590/S1806-37132014000100006

21. Akpinar EE, Hosgun D, Doganay B, Atac GK, Gulhan M, Should the cut-off value of D-dimer be elevated to exclude pulmonary embolism in acute exacerbation of COPD? J Thorac Dis. 2013;5(4):430-434. doi:10.3978/j.issn.2072-1439.2013.07.34

22. Bahloul M, Chaari A, Tounsi A, et al. Incidence and impact outcome of pulmonary embolism in critically ill patients with severe exacerbation of chronic obstructive pulmonary diseases. Clin Respir J. 2015;9(3):270-277.

23. Davoodi M, Rezvankhah B, Gohari Moghadam K, Hashemi Taheri AP. The prevalence and predicting factors of pulmonary thromboembolism in patients with exacerbated chronic obstructive pulmonary disease. Adv Respir Med. 2015. doi:10.5603/ARM. a2018.0025 
24. Gunen H, Gulbas G, In E, Yetkin O, Hacievliyagil SS, Venous thromboemboli and exacerbations of COPD. Eur Respir J. 2010;35 (6):1243-1248. doi:10.1183/09031936.00120909

25. Kamel MM, Moussa H, Ismail A, Prevalence of venous thrombo-embolism in acute exacerbations of chronic obstructive pulmonary disease. Egypt J Chest Dis Tuberc. 2013;62(4):557-566. doi:10.1016/j.ejcdt.2013.07.001

26. Rutschmann OT, Cornuz J, Poletti P-A, et al. Should pulmonary embolism be suspected in exacerbation of chronic obstructive pulmonary disease? Thorax. 2007;62(2):121-125.

27. Tillie-Leblond I, Marquette C-H, Perez T, et al. Pulmonary embolism in patients with unexplained exacerbation of chronic obstructive pulmonary disease: prevalence and risk factors. Ann Intern Med. 2006;144(6):390-396.

28. Tork M, Yildirim F, Sevimli N, Koktork N. Impact of pulmonary embolism on acute chronic obstructive pulmonary disease exacerbation. Gazi Med J. 2017;28(3):168-170.

29. Erelel M, Cuhadaro GC, Ece T, Arseven O, The frequency of deep venous thrombosis and pulmonary embolus in acute exacerbation of chronic obstructive pulmonary disease. Respir Med. 2002;96 (7):515-518. doi:10.1053/rmed.2002.1313

30. Hackx M, Ghaye B, Coche E, Van Muylem A, Gevenois PA, Severe COPD exacerbation: CT features. COPD J Chronic Obstr Pulm Dis. 2015;12(1):38-45. doi:10.3109/15412555.2014.903916

31. Cunningham DA, Lavender JP, Krypton $81 \mathrm{~m}$ ventilation scanning in chronic obstructive airways disease. $B r \quad J$ Radiol. 1981;54 (638):110-116. doi:10.1259/0007-1285-54-638-110

32. Johnson C, Connolly MJ, Clover S, et al. Audit of acute exacerbations of chronic obstructive pulmonary disease at Waitemata District Health Board, New Zealand. N Z Med J. 2013;126(1378).

33. Ozgun Niksarlioglu E, Aktürk Ü. Chest X-ray: is it still important in determining mortality in patients hospitalized due to chronic obstructive pulmonary diseases exacerbation in intensive care unit? Eurasian $J$ Pulmonol. 2018;20(3):133. doi:10.4103/ejop. ejop_70_18

34. Williams NP, Ostridge K, Devaster J-M, et al. Impact of radiologically stratified exacerbations: insights into pneumonia aetiology in COPD. Respir Res. 2018;19(1):143. doi:10.1186/s12931-0180842-8

35. Shafuddin E, Chang CL, Cooray M, et al. Cardiac dysfunction in exacerbations of chronic obstructive pulmonary disease is often not detected by electrocardiogram and chest radiographs. Intern Med J. 2019;49(6):761-769. doi:10.1111/imj.14144

36. Antenora F, Fantini R, Iattoni A, et al. Prevalence and outcomes of diaphragmatic dysfunction assessed by ultrasound technology during acute exacerbation of COPD: a pilot study. Respirology. 2017;22 (2):338-344.

37. Marchioni A, Castaniere I, Tonelli R, et al. Ultrasound-assessed diaphragmatic impairment is a predictor of outcomes in patients with acute exacerbation of chronic obstructive pulmonary disease undergoing noninvasive ventilation. Crit Care. 2018;22(1):109. doi:10.1186/s13054-018-2033-x

38. Saleh A, Lopez-Campos JL, Hartl S, Pozo-Rodriguez F, Roberts CM. The effect of incidental consolidation on management and outcomes in COPD exacerbations: data from the european COPD audit. PLoS One. 2015;10(7): 0134004.

39. Myint PK, Lowe D, Stone RA, Buckingham RJ, Roberts CM, Uk national COPD resources and outcomes project 2008: patients with chronic obstructive pulmonary disease exacerbations who present with radiological pneumonia have worse outcome compared to those with non-pneumonic chronic obstructive pulmonary disease exacerbations. Respiration. 2011;82(4):320-327. doi:10.1159/000327203

40. Hurst JR, Vestbo J, Anzueto A, et al. Susceptibility to exacerbation in chronic obstructive pulmonary disease. $N$ Engl J Med. 2010;363 (12):1128-1138. doi:10.1056/NEJMoa0909883
41. Wells JM, Washko GR, Han MK, et al. Pulmonary arterial enlargement and acute exacerbations of COPD. $N$ Engl J Med. 2012;367 (10):913-921. doi:10.1056/NEJMoa1203830

42. Selby C, Drost E, Lannan S, Wraith PK, Macnee W. Neutrophil retention in the lungs of patients with chronic obstructive pulmonary disease. Am Rev Respir Dis. 1991;143(6):1359-1364. doi:10.1164/ ajrccm/143.6.1359

43. Emerman CL, Cydulka RK, Evaluation of high-yield criteria for chest radiography in acute exacerbation of chronic obstructive pulmonary disease. Ann Emerg Med. 1993;22(4):680-684. doi:10.1016/ S0196-0644(05)81847-5

44. Feldman C, Richards GA, Smilg J. The findings on initial admission chest radiograph of patients presenting with an acute exacerbation of COPD - A South African Study. South African J Epidemiol Infect. 2015;30(2):57-60.

45. Fuso L, Incalzi RA, Pistelli R, et al. Predicting mortality of patients hospitalized for acutely exacerbated chronic obstructive pulmonary disease. Am J Med. 1995;98(3):272-277.

46. Hassen MF, Tilouche N, Jaoued O, Elatrous S. Incidence and impact of pulmonary embolism during severe COPD exacerbation. Respir Care. 2019;64:1531-1536. doi:10.4187/respcare.06661

47. Hoiseth AD, Omland T, Karlsson BD, Brekke PH, Soyseth V. Standardized evaluation of lung congestion during COPD exacerbation better identifies patients at risk of dying. Int $J$ COPD. 2013;8:621-629. doi:10.2147/COPD.S52854

48. Sherman S, Skoney JA, Ravikrishnan KP, Routine chest radiographs in exacerbations of chronic obstructive pulmonary disease. Diagnostic value. Arch Intern Med. 1989;149(11):2493-2496. doi:10.1001/archinte.1989.00390110077016

49. Sriram KB, Singh M, Lung ultrasound B-lines in exacerbations of chronic obstructive pulmonary disease. Intern Med J. 2017;47 (3):324-327. doi:10.1111/imj.13370

50. Titova E, Christensen A, Henriksen AH, Steinshamn S, Åsberg A. Comparison of procalcitonin, C-reactive protein, white blood cell count and clinical status in diagnosing pneumonia in patients hospitalized with acute exacerbations of COPD: a prospective observational study. Chron Respir Dis. 2019;16:147997231876976. doi: $10.1177 / 1479972318769762$

51. Park HJ, Kim SH, Kim H-C, et al. Utility of computed tomography in a differential diagnosis for the patients with an initial diagnosis of chronic obstructive pulmonary disease exacerbation. Tuberc Respir Dis. 2019;82(3):234. doi:10.4046/trd.2018.0087

52. Shapira-Rootman M, Beckerman M, Soimu U, Nachtigal A, Zeina A-R, The prevalence of pulmonary embolism among patients suffering from acute exacerbations of chronic obstructive pulmonary disease. Emerg Radiol. 2015;22(3):257-260. doi:10.1007/s10140-014-1280-7

53. Pizarro C, Herweg-Steffens N, Buchenroth $\mathrm{M}$, et al. Invasive coronary angiography in patients with acute exacerbated COPD and elevated plasma troponin. Int J COPD. 2016;11(1):2081-2089.

54. Guo X, Nie H, Chen Q, et al. The role of plasma N-terminal brain natriuretic pro-peptide in diagnosing elderly patients with acute exacerbation of COPD concurrent with left heart failure. Int J Chron Obstruct Pulmon Dis. 2018;13:2931-2940. doi:10.21 47/COPD.S164671

55. Lepida D, Papathanasiou A, Galiatsou E, Nakos G, Goudevenos I, Koulouras V. The contribution of left heart disease in COPD patients with pulmonary hypertension. Hell J Cardiol. 2018;59(3):160-165. doi:10.1016/J.HJC.2018.02.001

56. Lichtenstein D, Meziere G, A lung ultrasound sign allowing bedside distinction between pulmonary edema and COPD: the comet-tail artifact. Intensive Care Med. 1998;24(12):1331-1334. doi:10.1007/ s001340050771

57. Lim SY, Lim G, Lee YJ, et al. Ultrasound assessment of diaphragmatic function during acute exacerbation of chronic obstructive pulmonary disease: A pilot study. Int J COPD. 2019;14:2479-2484. doi:10.2147/COPD.S214716 
58. Mantuani D, Frazee BW, Fahimi J, Nagdev A, Point-of-care multi-organ ultrasound improves diagnostic accuracy in adults presenting to the emergency department with acute dyspnea. West J Emerg Med. 2016;17(1):46-53. doi:10.5811/westjem.2015.11.28525

59. Zechner PM, Aichinger G, Rigaud M, Wildner G, Prause G, Prehospital lung ultrasound in the distinction between pulmonary edema and exacerbation of chronic obstructive pulmonary disease. Am J Emerg Med. 2010;28(3):389. doi:10.1016/j.ajem.2009.07.021
60. Cukic V, Begic A, Potential role of lung ventilation scintigraphy in the assessment of COPD. Acta Inform Medica. 2014;22(3):170-173. doi:10.5455/aim.2014.22.170-173

61. Bing D, Jian K, Long-feng S, Wei T, Hong-wen Z, Vibration response imaging: A novel noninvasive tool for evaluating the initial therapeutic effect of noninvasive positive pressure ventilation in patients with acute exacerbation of chronic obstructive pulmonary disease. Respir Res. 2012;13(1):65. doi:10.1186/1465-9921-13-65

\section{Publish your work in this journal}

The International Journal of COPD is an international, peer-reviewed journal of therapeutics and pharmacology focusing on concise rapid reporting of clinical studies and reviews in COPD. Special focus is given to the pathophysiological processes underlying the disease, intervention programs, patient focused education, and self management protocols. This journal is indexed on PubMed Central, MedLine and CAS. The manuscript management system is completely online and includes a very quick and fair peer-review system, which is all easy to use. Visit http://www.dovepress.com/testimonials.php to read real quotes from published authors. 